\title{
Clustering on very small scales from a large sample of confirmed quasar pairs: does quasar clustering track from Mpc to kpc scales?
}

\author{
S. Eftekharzadeh, ${ }^{1 \star}$ A. D. Myers, ${ }^{1}$ J. F. Hennawi, ${ }^{2,3}$ S. G. Djorgovski, ${ }^{4}$ \\ G. T. Richards, ${ }^{5}$ A. A. Mahabal ${ }^{6}$ and M. J. Graham ${ }^{4,7}$ \\ ${ }^{1}$ Department of Physics and Astronomy, University of Wyoming, 1000 University Ave, Laramie, WY 82071, USA \\ ${ }^{2}$ Max-Planck-Institut fur Astronomie, Königstuhl 17, D-69117 Heidelberg, Germany \\ ${ }^{3}$ Department of Physics, Broida Hall, University of California, Santa Barbara, CA 93106-9530, USA \\ ${ }^{4}$ Astronomy Department, California Institute of Technology, 1200 East California Boulevard, Pasadena, CA 91125, USA \\ ${ }^{5}$ School of Physics and Center for Relativistic Astrophysics, Georgia Institute of Technology, 837 State Street NW, Atlanta, GA 30332-043, USA \\ ${ }^{6}$ Cahill Center for Astronomy and Astrophysics, California Institute of Technology, 1200 E California Blvd, MC 249-17, Pasadena, CA 91125, USA \\ ${ }^{7}$ National Optical Astronomy Observatory, 950 N Cherry Avenue, Tucson, AZ 85719, USA
}

Accepted 2017 February 14. Received 2017 February 9; in original form 2017 January 29

\begin{abstract}
We present the most precise estimate to date of the clustering of quasars on very small scales, based on a sample of 47 binary quasars with magnitudes of $g<20.85$ and proper transverse separations of $\sim 25 \mathrm{~h}^{-1} \mathrm{kpc}$. Our sample of binary quasars, which is about six times larger than any previous spectroscopically confirmed sample on these scales, is targeted using a kernel density estimation (KDE) technique applied to Sloan Digital Sky Survey (SDSS) imaging over most of the SDSS area. Our sample is 'complete' in that all of the KDE target pairs with $17.0 \lesssim R \lesssim 36.2 h^{-1} \mathrm{kpc}$ in our area of interest have been spectroscopically confirmed from a combination of previous surveys and our own long-slit observational campaign. We catalogue 230 candidate quasar pairs with angular separations of $<8$ arcsec, from which our binary quasars were identified. We determine the projected correlation function of quasars $\left(\bar{W}_{\mathrm{p}}\right)$ in four bins of proper transverse scale over the range $17.0 \lesssim R \lesssim 36.2 h^{-1} \mathrm{kpc}$. The implied small-scale quasar clustering amplitude from the projected correlation function, integrated across our entire redshift range, is $A=24.1 \pm 3.6$ at $\sim 26.6 h^{-1} \mathrm{kpc}$. Our sample is the first spectroscopically confirmed sample of quasar pairs that is sufficiently large to study how quasar clustering evolves with redshift at $\sim 25 h^{-1} \mathrm{kpc}$. We find that empirical descriptions of how quasar clustering evolves with redshift at $\sim 25 h^{-1}$ Mpc also adequately describe the evolution of quasar clustering at $\sim 25 h^{-1} \mathrm{kpc}$.
\end{abstract}

Key words: surveys-quasars: general-cosmology: observations-large-scale structure of universe.

\section{INTRODUCTION}

Quasars, like galaxies, are biased tracers of the underlying dark matter distribution (e.g. Cole \& Kaiser 1989; Berlind \& Weinberg 2002). Many models invoke galaxy mergers as the mechanism for triggering quasar activity, although the necessity of such a mechanism is still debated (e.g. Coil et al. 2007; Padmanabhan et al. 2009; Green et al. 2011). Certainly, though, structure formation models can reproduce quasar demographics under the assumption that quasar activity is triggered by mergers (e.g. Wyithe \& Loeb 2005). The peaks of the density field in which quasars reside might have been particularly strongly clustered, given that mergers

^E-mail: seftekha@uwyo.edu. are more frequent in denser environments (e.g. Kaiser 1984; Lacey \& Cole 1993; Di Matteo, Springel \& Hernquist 2005; Hopkins et al. 2008) and that density signals from mergers can persist on time-scales similar to the lifetime of quasars (Wetzel et al. 2009). Quasar clustering measurements therefore offer a tool by which to understand the physical processes that trigger quasar activity. The ongoing attempts to conduct such investigations become more challenging at higher luminosities (e.g. Ellison et al. 2011, 2013; Jiang et al. 2016).

Surveys such as the Sloan Digital Sky Survey (SDSS) increased the sample size and number density of quasars in a large volume of space, substantially improving measurements of quasar clustering on large or 'two-halo' scales (e.g. Porciani, Magliocchetti \& Norberg 2004; Croom et al. 2005; Myers et al. 2006, 2007a; Porciani \& Norberg 2006; Shen et al. 2007, 2009; Ross et al. 2009; 
White et al. 2012; Eftekharzadeh et al. 2015). Measuring quasar clustering on small scales, however, is more challenging for several reasons. First, quasars with small angular separations $(<60$ arcsec) are simply rare. Secondly, surveys that use fibre-fed multi-object spectrographs, such as the SDSS, prevent fibres from colliding by never placing two fibres closer than about 60 arcsec on a single plate (Blanton et al. 2003; Dawson et al. 2013). Thirdly, finding rare quasar pairs without exploiting large surveys typically requires many individual long-slit observations of pairs of candidates, which is time-consuming.

The first small-scale quasar pairs were often discovered by chance in dedicated fields, or during long-slit surveys for gravitationally lensed quasars ${ }^{1}$ (e.g. Sramek \& Weedman 1978; Weedman et al. 1982; Crampton et al. 1988; Hewett et al. 1989; Meylan \& Djorgovski 1989; Schneider, Schmidt \& Gunn 1994; Hagen et al. 1996; Fan et al. 1999; Kochanek, Falco \& Muñoz 1999; Mortlock, Webster \& Francis 1999; Schneider et al. 2000; Gregg et al. 2002; Miller et al. 2004; Pindor et al. 2006; McGreer et al. 2016). Although the search for high-redshift quasar pairs dates back to individual discoveries of quasars at $z \sim 4$ (e.g. Crampton et al. 1988; McCarthy et al. 1988; Meylan et al. 1990; Djorgovski 1991; Schneider et al. 1994; Hewett et al. 1998; Zhdanov \& Surdej 2001), with the development of photometric selection algorithms to build homogeneous sets of quasar candidates from large imaging surveys (e.g. Richards et al. 2004, 2009), it became possible to conduct more homogeneous searches by prioritizing highly probable close quasar pairs and following them up with long-slit spectroscopic surveys (e.g. Hennawi et al. 2006a; Myers et al. 2007b, 2008). These surveys focused on quasar pairs separated by less than $2000 \mathrm{~km} \mathrm{~s}^{-1}$ in redshift space in order to measure small-scale clustering, denoting such pairs 'binary quasars', a term that has appeared for decades in the literature (e.g. Muñoz et al. 1998). Hennawi et al. (2006a) elucidate the specific use of a velocity range of $|\Delta v|<2000 \mathrm{~km} \mathrm{~s}^{-1}$ as being wide enough to cover the most prominent sources of redshift uncertainty for quasars, in particular peculiar velocities of up to $500 \mathrm{~km} \mathrm{~s}^{-1}$ in dense environments and blueshifted broad lines of up to $1500 \mathrm{~km} \mathrm{~s}^{-1}$ (Richards et al. 2002; Hennawi et al. 2006a).

In tandem with similar homogeneous searches for gravitational lenses (Oguri et al. 2006, 2008, 2012; Inada et al. 2008, 2010, 2012), work on binary quasars has driven measurements of quasar clustering on very small scales down below even a few hundred kiloparsecs ( $\sim 10$ arcsec or lower). For example, Kayo \& Oguri (2012) took advantage of the SDSS Quasar Lens Search (Inada et al. 2012) to measure the quasar correlation function down to $\sim 10 h^{-1} \mathrm{kpc}$.

In this paper, we continue in the vein of Hennawi et al. (2006a) and Myers et al. (2007b, 2008). We identify high-probability candidate close quasar pairs from a homogeneous catalogue of candidates and follow them up with confirming spectroscopy. Our target sample is drawn from quasar candidates selected using kernel density estimation (KDE) by Richards et al. (2009). This 'KDE' sample is not only large, but also pure, ${ }^{2}$ so it presents an efficient parent sample to mine for binary quasars. The sample of 47 confirmed binary quasars that we will discuss in this paper is complete for

\footnotetext{
${ }^{1}$ SDSS J1637+2636AB was the first binary quasar discovered, but originally misinterpreted as a lens (Djorgovski \& Spinrad 1984). Due to this misinterpretation, the quasar pair PKS 1145-071 was initially known as the first binary quasar (Djorgovski et al. 1987).

${ }^{2}>90$ per cent of $\mathrm{KDE}$ candidates at $0.4 \lesssim z \lesssim 2.3$ are expected to be quasars.
}

angular separations of 2.9 arcsec $<\theta<6.3$ arcsec and redshifts of $0.43<z<2.26$. Note that we will use the term 'complete' here to refer to 100 per cent confirmation of whether or not all of our candidate target pairs are binary quasars. We do not mean complete in the sense of also capturing quasars that are not in the KDE catalogue. Our sample improves on previous work in being over five times larger than previous samples of binaries on the range of scales that we cover $\left(\sim 20-40 h^{-1} \mathrm{kpc}\right)$. The $>2 \times$ more precise correlation function that we calculate over proper separations at $<50 h^{-1} \mathrm{kpc}$ is therefore the tightest constraint on quasar clustering to date on scales of a few tens of kiloparsecs.

This paper is structured as follows. The data are introduced in Section 2, and our methodology for measuring and modelling clustering is discussed in Section 3.1. Section 4 is dedicated to the interpretation of our clustering results, before we summarize our work in Section 5. We adopt a $\Lambda$ cold dark matter cosmological model with $\Omega_{\mathrm{m}}=0.307, \Omega_{\Lambda}=0.693$ and $h=0.677$ consistent with Planck Collaboration XIII (2016). All distances quoted throughout the paper are in proper coordinates unless mentioned otherwise. We convert measurements from the literature to proper coordinates prior to comparing such measurements to our results. We use 'cMpc' and 'ckpc' to denote comoving distance units when we compare our measurements in proper coordinates to correlation lengths in comoving coordinates that have been derived from Mpc-scale clustering measurements. In our chosen cosmology, an angular separation of $1 \operatorname{arcsec}$ at $z=1.5$ corresponds to a proper separation of $5.9 h^{-1} \mathrm{kpc}$.

\section{DATA}

\subsection{Identification of new quasar pairs}

Our starting sample consists of 1172157 high-probability candidate quasars identified by Richards et al. (2009) using KDE (see also Richards et al. 2004). Richards et al. (2009) applied the KDE technique to a test sample consisting of all point sources in SDSS Data Release 6 (DR6; Adelman-McCarthy et al. 2008) imaging down to a limiting magnitude of $i=21.3$. These test data were labelled as a 'star' or a 'quasar' using a non-parametric Bayesian classifier, based on their position in ugriz colour space. The point spread function magnitudes of the sources were extinction-corrected based on the Schlegel, Finkbeiner \& Davis (1998) dust maps. The density of the 'quasar' and 'star' colour space was established by applying the KDE technique to 'training' samples of stars and quasars. The 'stars' training set resembled a randomly drawn subset of the test set. The 'quasars' training set consisted of spectroscopically confirmed SDSS quasars (Schneider et al. 2007) largely limited to $i<19.1$ at $z<3$ and $i<20.2$ at $z>3$. At higher redshift, the quasar training sample was supplemented by quasars from the AAOmega-UKIDSS-SDSS (AUS) QSO survey (Croom et al., in preparation) and from Fan et al. (2006). Given that the position of the quasar locus in colour space relative to that of the stellar locus changes significantly with redshift, Richards et al. (2009) conducted a redshift-and-colour-based subclassification in four narrower ranges of low redshift $(z \leq 2.2)$; intermediate redshift (2.2 $\leq z \leq 3.5$ ); high redshift ( $z \geq 3.5$ ); and also UV-excess (UVX), based on $u-g$ colour. High-probability quasars classified in these ranges are denoted as lowzt $s==1$, midzt $s==1$, hizts $==1$ or uvxts $==1$, respectively (see table 2 of Richards et al. 2009).

From the initial KDE sample of 1172157 candidate quasars, we subselected candidates that are brighter than 20.85 in (Galacticextinction-corrected) $g$ band and are categorized as hizts $==1$ 
Table 1. Summary of the follow-up spectroscopic campaign for a complete subsample of the KDE-selected quasar candidates. The Reference column refers to (1) Sheinis et al. (2002) and (2) Oke \& Gunn (1983).

\begin{tabular}{|c|c|c|c|c|c|c|}
\hline Telescope & Instrument & $\begin{array}{l}\text { Spectrograph } \\
\text { type }\end{array}$ & $\begin{array}{c}\text { Spectral } \\
\text { coverage }(\AA)\end{array}$ & FWHM & Dates & Reference \\
\hline Mayall $4 \mathrm{~m}$ & Ritchey-Chrétien spectrograph (RC) & Single & $3600-9200$ & 325 & 2008 Feb. 9-11, Jun. 7-10 & (1) \\
\hline Palomar 200 inch & Double spectrograph & Double & $3100-9300$ & $900 / 550$ & 2009 Feb. 24, Mar. 30, Apr. 27 \& Jun. 17 & (2) \\
\hline Palomar 200 inch & Double spectrograph & Double & $3100-9300$ & $900 / 550$ & 2000 Nov. $7-10$ & (2) \\
\hline Palomar 200 inch & Double spectrograph & Double & $3100-9300$ & $900 / 550$ & 2011 Mar. 2-3 & (2) \\
\hline
\end{tabular}

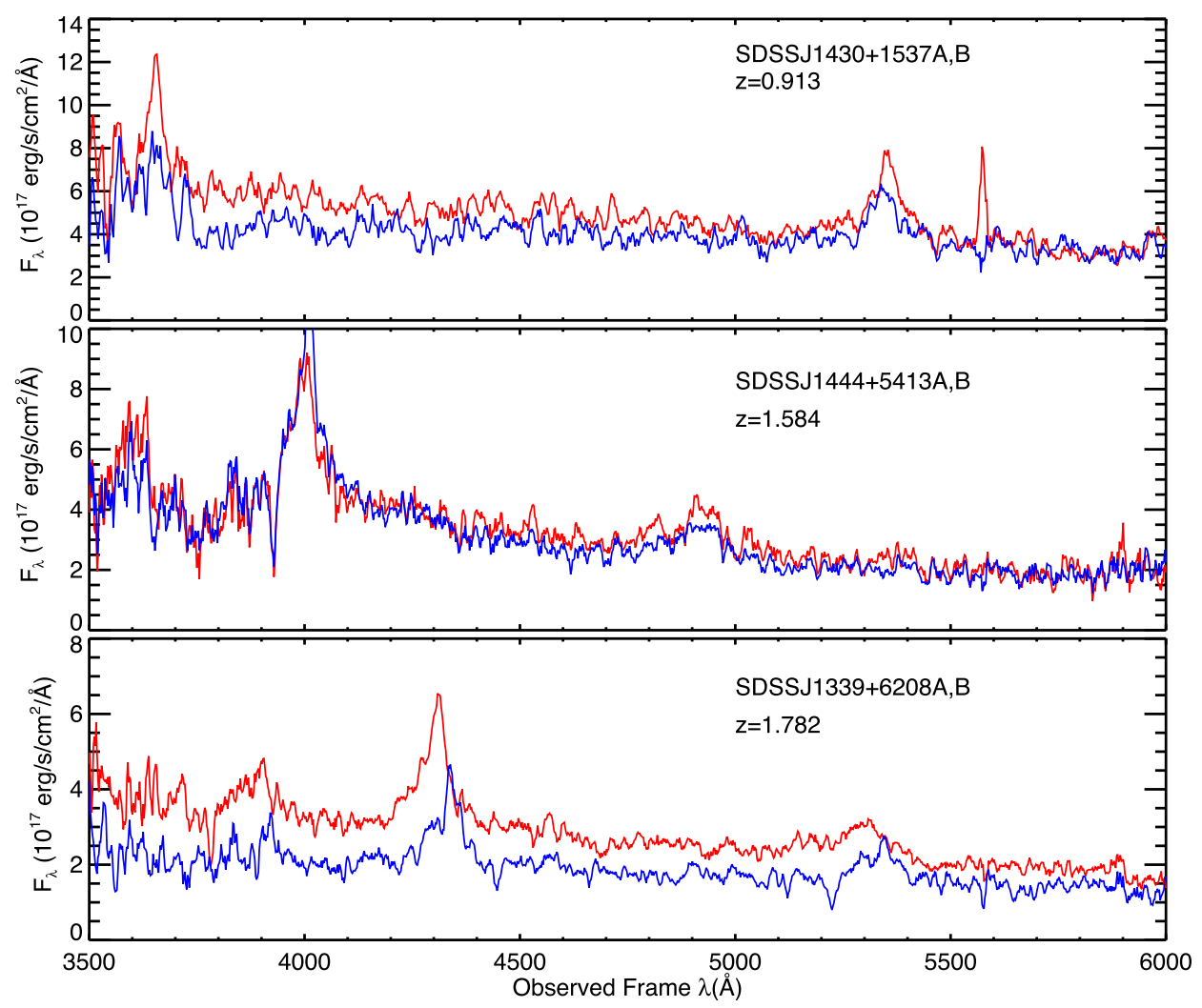

Figure 1. Three example spectra of confirmed binary quasars. The spectra are smoothed by 5 pixels to aid visualization.

OR uvxts $==1$. We further restricted this subsample to the $70^{\circ}<\mathrm{RA}<300^{\circ}$ region of the DR6 imaging footprint, resulting in a total of 369559 quasar candidates. We will hereafter refer to these 369559 candidates as our 'parent sample'. We cross-matched the candidates by angular separation and identified 230 candidate pairs with separations of $2.8 \operatorname{arcsec}<\theta<8$ arcsec. Here, the upper limit is chosen to correspond to a few hundred kpc for likely quasar redshifts. The lower limit is chosen to match roughly twice the seeing of the SDSS imaging data (e.g. see fig. 4 of Abazajian et al. 2003) in order to protect against sources that are merged in SDSS imaging.

To determine which of our candidate pairs had already been identified as quasars, we used a radius of 1 arcsec to cross-match our parent sample with previously known spectroscopically confirmed, visually inspected quasars. These 'known' quasars were drawn from programmes conducted to identify quasar pairs (Hennawi et al. 2006a,b; Myers et al. 2008; Hennawi et al. 2010; Hennawi \& Prochaska 2013; Prochaska et al. 2013; Prochaska, Lau \&
Hennawi 2014) and gravitational lenses (Inada et al. 2008, 2012; Oguri et al. 2008, 2012) as well as SDSS Data Release 7 (DR7; Schneider et al. 2010) and Data Release 12 (DR12; Pâris et al. 2016, henceforth DR12Q). In particular, DR12Q includes objects from an SDSS ancillary programme designed specifically to target some of our candidate quasars. ${ }^{3}$ We then identified candidate quasar pairs that did not have both members of the pair previously spectroscopically confirmed and reserved such $(2.8 \operatorname{arcsec}<\theta<8$ arcsec $)$ pairs for further spectroscopic confirmation.

Long-slit spectroscopy of these selected candidate quasar pairs was conducted on a range of facilities outlined in Table 1, with the slit oriented to observe both quasars simultaneously. The spectra were reduced and calibrated using the XIDL package. ${ }^{4}$ Figs 1 and 2 show three examples of reduced spectra of our quasar pairs.

\footnotetext{
${ }^{3}$ http://www.sdss.org/dr12/algorithms/ancillary/boss/smallscaleqso/

${ }^{4}$ http://www.ucolick.org/xavier/IDL/index.html
} 


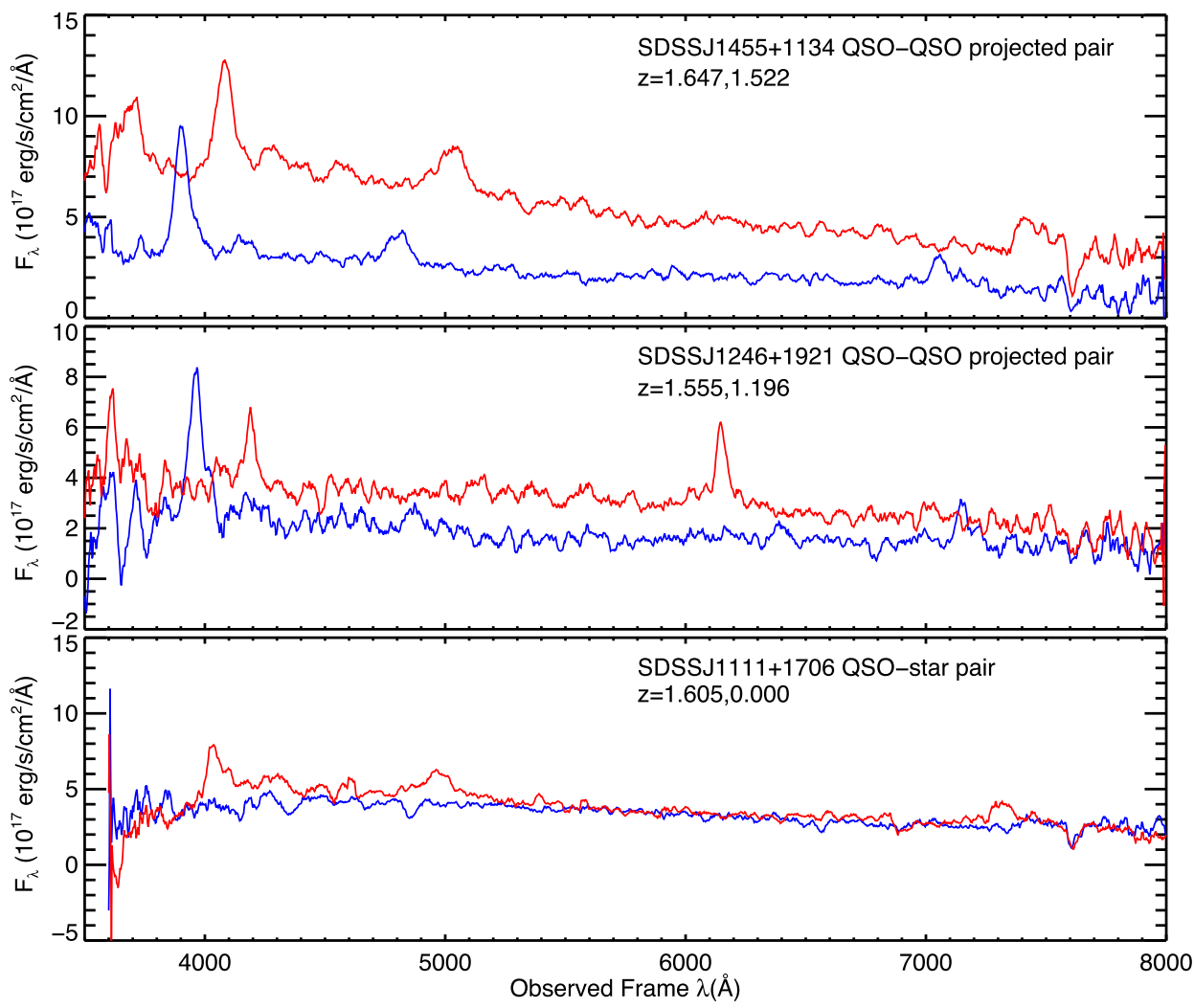

Figure 2. Three example spectra of quasar pairs that are not binaries. Such pairs can be two quasars that are aligned along the line of sight but have different redshifts, star-quasar pairs or star-star pairs.

\subsection{A KDE-complete sample of binary quasars}

Our goal is to characterize quasar clustering on very small scales using a statistically uniform sample of quasars that are proximate to each other (so-called binary quasars). Following Hennawi et al. (2006a), we designate pairs of quasars that do not meet this criterion to be 'projected pairs'. Over the course of our campaign to date, we have obtained definitive classifications for a close to complete sample of KDE-selected candidates on angular scales of $2.9 \operatorname{arcsec} \leq \theta \leq 7.7$ arcsec, which correspond to proper scales ${ }^{5}$ of roughly $15 \leq R \leq 40 h^{-1} \mathrm{kpc}$ over the main redshift range of our sample. The resulting sample consists of 169 candidate quasar pairs, which we will refer to as our sample of 'relevant pairs'. Note that good spectroscopy of both candidates is not required to 'definitively classify' a pair as not a binary quasar. For instance, if one of a pair of objects is categorically identified as a star or a galaxy, then that pair is a non-binary, and we classify it as a 'projected pair'. Further, if a known quasar at a redshift of $z$ has a companion for which we have a spectrum that is of sufficiently high quality that we should certainly have identified broad emission lines corresponding to $z$, then we also classify that pair as a projected pair. Note that we do not consider confirmed quasar pairs to be 'binary' even if their velocity separation is only slightly larger than $2000 \mathrm{~km} \mathrm{~s}^{-1}$. In addition, we removed one pair ${ }^{6}$ from our 'relevant pair' sample that consisted of two high signal-to-noise but featureless ('continuum') sources.

\footnotetext{
${ }^{5}$ We use the angular separation and confirmed redshift of the brighter member in each of our relevant pairs to calculate the proper transverse separation between members of the pair.

${ }^{6}$ SDSS J1336+2737 with a separation of 5.41 arcsec.
}

Table 2. Classification of all 169 'relevant pairs' in our sample (with $g<20.85$ and $2.9 \operatorname{arcsec}<\Delta \theta<7.7$ arcsec). 'Confirmed binaries' meet the classification of a binary quasar for the purposes of this paper $\left(\left|\Delta v_{\|}\right|<2000 \mathrm{~km} \mathrm{~s}^{-1}\right.$ and not otherwise identified as a gravitational lens); 'confirmed quasar pairs' denotes pairs for which we have spectroscopic information for both members of the candidate pair and that have $\left|\Delta v_{\|}\right| \geq 2000 \mathrm{~km} \mathrm{~s}^{-1}$.

\begin{tabular}{lc}
\hline Category & \# of pairs \\
\hline Confirmed binaries & 58 \\
Confirmed lenses & 5 \\
Confirmed quasar pairs (non-binaries) & 77 \\
Pairs with at least one confirmed non-quasar member & 8 \\
Pairs with at least one unknown member & 21 \\
\hline
\end{tabular}

Even if this pair is a binary quasar, we would have no way to assign it a redshift.

Table 2 records the nature of our total of 169 relevant pairs, including their ultimate classification as a binary quasar, a projected pair, a pair for which there is insufficient information to characterize it or a gravitational lens. ${ }^{7}$ The distribution on the sky of the binary quasars in our sample of relevant pairs is shown in Fig. 3, against a background of all of the KDE-selected candidates in our parent sample. (See Table 3 for the normalized distribution of their spectroscopic redshifts.) Our follow-up spectroscopy of candidate pairs provided 126 new sets of observations of candidate quasar pairs that have separations of less than 7.7 arcsec. Of these 126 newly

\footnotetext{
${ }^{7}$ We designate binary quasars as gravitational lenses if they are convincingly argued to be lenses in the literature.
} 
Table 3. Normalized distribution of the spectroscopic redshifts of quasars in our parent sample of candidates. The full table is available in the electronic version of this paper.

\begin{tabular}{cc}
\hline$\Delta z$ & $(1 / N) \mathrm{d} N / \mathrm{d} z$ \\
\hline 0.43 & 0.191 \\
0.44 & 0.239 \\
0.45 & 0.200 \\
0.46 & 0.230 \\
0.47 & 0.240 \\
0.48 & 0.225 \\
0.49 & 0.239 \\
0.50 & 0.284 \\
0.51 & 0.288 \\
0.52 & 0.288 \\
0.53 & 0.305 \\
0.54 & 0.308 \\
0.55 & 0.258 \\
0.56 & 0.339 \\
0.57 & 0.303 \\
0.58 & 0.322 \\
0.59 & 0.389 \\
0.60 & 0.366 \\
0.61 & 0.398 \\
0.62 & 0.385 \\
\hline
\end{tabular}

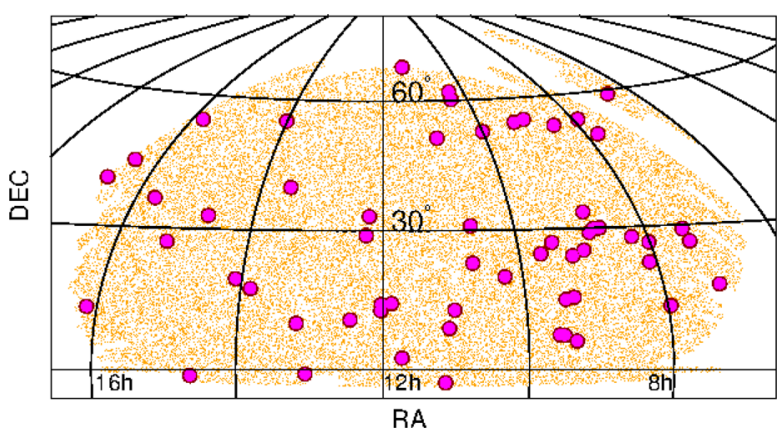

Figure 3. Coordinates of the 369559 quasar candidates in our parent sample (orange) in Aitoff projection. The filled circles depict the 58 spectroscopically confirmed binaries in our sample of relevant pairs. The data have been cut to the NGC imaging footprint of SDSS DR6, our main area of focus.

characterized pairs, we confirmed 53 to be binary quasars. Richards et al. (2009) used clustering analyses to estimate that the KDE selection algorithm is 92.7 per cent efficient for sources with $\mathrm{hi} z \mathrm{t} \mathrm{s}==1$ OR uvxt $s==1$. If we designate 'stars' as those objects in our sample that do not have a sufficiently good spectrum to classify the object, ${ }^{8}$ then we find that out of the 338 sets of candidate quasars in our sample of 169 relevant pairs, we confirm 309 to be quasars. This is in excellent agreement with an efficiency of $\sim 92.5$ per cent for the KDE catalogue.

Typically, clustering studies construct a random catalogue, or otherwise analytically correct for sources of incompleteness that arise when targeting quasars (e.g. equation 17 of Hennawi et al. 2006a). To circumvent incompleteness corrections when conducting clustering analyses, we instead construct a sample of pairs that we have categorically identified as either binary quasars or not. We

\footnotetext{
${ }^{8}$ A reasonable assumption, given that quasars are much easier to classify at low signal to noise as compared to stars.
}

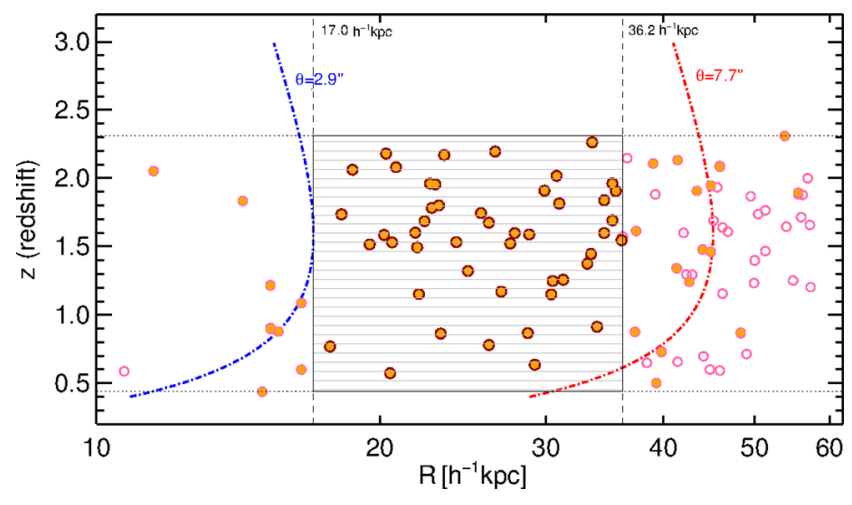

Figure 4. Redshift and proper transverse separation range probed by the binary quasars in our 'KDE-complete' sample. Filled circles represent spectroscopically confirmed binary quasars. Open circles represent binary quasars for which spectroscopic information exists for only one of the members of the pair. The dotted lines depict transverse separations corresponding to $2.9 \operatorname{arcsec} \leq \theta \leq 7.7$ arcsec, the angular range of our 169 relevant candidate quasar pairs. The extent of our 'KDE-complete' sample of 47 binaries is depicted by a grey box that is limited by either the angular extent of our sample of relevant pairs or quasar pairs that we cannot currently confirm or reject as binary based on spectroscopy.

will henceforth refer to this subset of pairs as our 'KDE-complete' sample. Binary quasars in the KDE-complete redshift and properscale ranges can be used for clustering analyses without correcting for incompleteness in our spectroscopic campaign (because, by definition, this range is 100 per cent complete to possible binary quasars in our parent sample). The outer limits of our KDE-complete sample in redshift and proper transverse scale are defined by the ranges at which there exist quasar pairs that we cannot categorically classify as binary or not. Typically, this is because the spectroscopic information for either one or both members of the pair does not exist. Note that there are cases where spectroscopic confirmation of only one member of a pair is sufficient to include that pair in the KDE-complete sample. Most obviously, as also noted above, pairs that include one non-quasar have sufficient information to be included in the KDE-complete sample. In addition, though, pairs that include one confirmed quasar with a (spectroscopic) redshift that would categorically place it outside of the proper-scale range of interest can also be used to define the KDE-complete ranges, regardless of whether such a quasar's companion has itself been spectroscopically confirmed.

Fig. 4 shows the redshift and proper transverse separation ranges for binary quasars in our KDE-complete sample. The dotted lines show the transverse separations corresponding to the $2.9 \operatorname{arcsec} \leq$ $\theta \leq 7.7$ arcsec angular range of our 169 relevant pairs. The extent of the KDE-complete sample of binaries both in redshift and in transverse separation is depicted by a grey box. This box is limited by either the angular extent of our sample of relevant pairs or quasar pairs that we cannot currently confirm or reject as binary based on the spectroscopic information to hand (depicted by open circles in Fig. 4). The ranges of redshift and proper separation that define the limits of the KDE-complete sample (i.e. the edges of the grey box in Fig. 4) are $0.44 \leq z \leq 2.31$ and $17.0 \leq R \leq 36.2 h^{-1}$ kpc. Fig. 4 also illustrates that we only consider a small fraction of space with $\theta<3$ arcsec, in keeping with the arguments in Pindor et al. (2003) and Hennawi et al. (2006a) that sources with $\theta<3 \operatorname{arcsec}$ can appear blended in SDSS imaging. 


\section{METHODOLOGY}

\subsection{Estimating the small-scale clustering of quasars}

We measure the correlation function in proper coordinates, projected across a redshift window of $<2000 \mathrm{~km} \mathrm{~s}^{-1}$ (our definition of a 'binary quasar' from Section 2), using the estimator

$\bar{W}_{\mathrm{p}}=\frac{Q Q}{\langle Q R\rangle}-1$.

(e.g. Peebles 1973; Shanks et al. 1987; Croom \& Shanks 1996). Here, $Q Q$ represents a count of quasar-quasar data pairs and $\langle Q R\rangle$ denotes the 'expected' number of quasar-random pairs in a given bin of redshift, angle or proper separation. Note that

$\langle Q R\rangle=\frac{N_{\mathrm{Q}}}{N_{\mathrm{R}}} Q R$,

where $N_{\mathrm{Q}} / N_{\mathrm{R}}$ is the size of the quasar catalogue compared to the size of a (larger) random catalogue. An appropriate random catalogue will mimic the angular and redshift distribution of the data in the absence of any clustering. Since our KDE-complete sample of binary quasars is drawn from the KDE catalogue described in Section 2, the random catalogue needs to have the same overall angular and redshift coverage as the KDE catalogue (see, e.g. Myers et al. 2006, 2007a).

The entire volume of the KDE catalogue comprises $\sim 41.93\left(h^{-1} \mathrm{Gpc}\right)^{3}$. Generating a sufficiently large random catalogue over such a volume purely for the purposes of making a kpc-scale clustering measurement is a computationally expensive task. Such an approach is also unnecessary, as we only seek $Q R$ pairs with small angular separations ( $\leq 7.7$ arcsec). We therefore construct a random catalogue for our analysis using three independent steps. As our sample of pairs is complete for proper scales of $17.0 \leq R \leq 36.2 h^{-1} \mathrm{kpc}$ (see Section 2), these three steps are sufficient to model the expected unclustered distribution of our sample of binary quasars.

(1) We randomly selected a subset of $N_{\mathrm{Q}}=342581 \mathrm{KDE}$ candidate quasars, corresponding to 92.7 per cent of our parent sample of $369559 \mathrm{KDE}$ candidates (see Section 2). This down-sampling is necessary because the efficiency of the KDE algorithm for selecting our overall sample of candidate quasars (lowzts and uvxts; again see Section 2) is $\sim 92.7$ per cent.

We randomly generated positions around these $342581 \mathrm{KDE}$ candidate quasars on angular scales of $2.9 \operatorname{arcsec}<\theta<7.7$ arcsec, which is the range of angular separations of candidate quasar pairs on our 'KDE-complete' scales of interest (see Section 2 and specifically Fig. 4). We will refer to the resulting catalogue as our angular random catalogue.

(2) Only $\sim 36$ per cent (131 928) of the KDE candidates have a confirmed spectroscopic redshift. We used the full distribution of spectroscopic redshifts in the KDE sample, displayed in Fig. 5, and randomly drew redshifts from the resulting $\mathrm{d} N / \mathrm{d} z$ both for those candidates with no spectroscopic redshift and for objects in our angular random catalogue. Then, working with quasars with redshifts within our range of interest $(0.43<z<2.26)$, we downsampled our angular random catalogue by retaining only random points in $Q R$ pairs separated by $<2000 \mathrm{~km} \mathrm{~s}^{-1}$ (our definition of a 'binary quasar' from Section 2). We will refer to the resulting catalogue as our redshift random catalogue.

(3) Using the redshift and angular separation information that we generated in steps (1) and (2), we further limited our redshift random catalogue to only $Q R$ pairs that intersected with the limits in redshift and proper scale of our 'KDE-complete' sample of binary

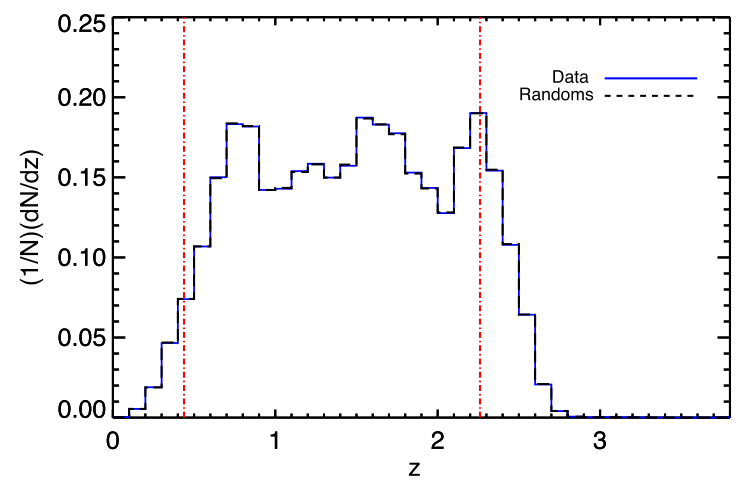

Figure 5. The normalized redshift distribution of spectroscopically confirmed quasars in our sample of relevant pairs (blue solid line) compared to the generated distribution for our redshift random catalogue (black dashed line). The vertical red dot-dashed lines delineate the redshift range of the KDE-complete sample.

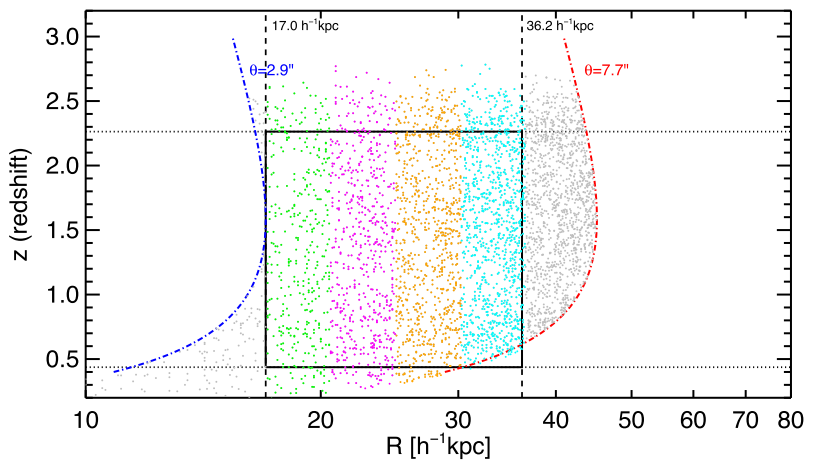

Figure 6. The bins in proper scale that we use in our clustering measurement are shown in different colours. This figure illustrates the difference between how the random catalogue is populated in angle and the resulting random points that are counted in bins of proper scale. The black box represents the limits of our KDE-complete sample (see also Fig. 4).

quasars (see Fig. 6). We will refer to the resulting catalogue as our final random catalogue. A total of $290694 \mathrm{KDE}$ candidate quasars have spectroscopic redshifts in the range of our complete sample of binary quasars $(0.43<z<2.2)$. The final random catalogue (' $R$ ') can be used in conjunction with these $290694 \mathrm{KDE}$ candidate quasars (' $Q$ ') to calculate $Q R$ in equation (2) as a function of scale or redshift.

The steps that produce the redshift and final random catalogues discard points that do not create eligible $Q R$ pairs. It is therefore necessary to generate a large enough initial angular random catalogue to retain a sufficiently large final random catalogue with which to infer $\langle Q R\rangle$. We found that assigning each of the 290694 KDE candidate quasars in our redshift range of interest $N=2000$ random points on scales of $0 \operatorname{arcsec}<\theta<7.7 \operatorname{arcsec}^{9}$ was sufficient in this regard, as such a schema ultimately provided more than 20 random points around each KDE candidate quasar. Essentially, this means that our final random catalogue is at least $20 \times$ larger than our data catalogue.

An important consideration is that the $N_{\mathrm{R}}$ in equation (2) does not denote the $N=2000$ random points that we generated around each

\footnotetext{
${ }^{9}$ We assigned 2000 points over $\theta<7.7$ arcsec and then clipped them to cover $2.9 \operatorname{arcsec}<\theta<7.7$ arcsec to provide flexibility if our minimum angle changed.
} 
of the $N_{\mathrm{Q}}=290694 \mathrm{KDE}$ candidate quasars in our redshift range of interest. Rather, it corresponds to the number of random points that would have truly been generated had we chosen to populate the entire survey volume. We calculate $N_{\mathrm{R}}$ as the 'populated areal number density of the random points' $x$ 'the full area of the survey footprint':

$N_{R}=\frac{N}{A(<7.7 \operatorname{arcsec})} A_{\text {full }}$,

where $N=2000$ is the number of random points we generated around each candidate quasar to $\theta<7.7 \operatorname{arcsec}, A(<7.7 \operatorname{arcsec})$ is the survey area within 7.7 arcsec of a candidate quasar and $A_{\text {full }}$ is the full area of the survey footprint (the orange footprint in Fig. 3).

To calculate the survey area, we use the SDSS 'survey coordinates', $\eta$ and $\lambda$ (e.g. Stoughton et al. 2002), to construct stripeshaped polygons along great circles using the MANGLE software (Blanton et al. 2003; Tegmark et al. 2004; Swanson et al. 2008). We also create 'holes' in the footprint corresponding to SDSS imaging masks. ${ }^{10}$ Note that when we created the angular random catalogue, we discarded any points that lay in holes or outside of the survey area, but this made very little difference on scales of $\theta<7.7$ arcsec. Based on this process, the total area of the survey footprint that is used in this study is $A_{\text {full }}=7600.4 \mathrm{deg}^{2}$. Since we only consider angular scales up to 7.7 arcsec, the 'effective' area around any individual candidate quasar is $A(<7.7$ arcsec $)=1.44 \times 10^{-5} \mathrm{deg}^{2}$. So, $N_{\mathrm{R}}=(2000 \times 7600.4) / 1.44 \times 10^{-5} \sim 10^{12}$. In other words, the process that we have outlined would be equivalent to generating a very, very large random catalogue across the entire survey volume.

\subsection{Theoretical considerations}

The volume-averaged projected correlation function $\left(\bar{W}_{\mathrm{p}}\right)$ is a useful estimator for our purposes given the large volume occupied by quasars over a wide redshift range, compared to the small scales on which we seek to measure clustering. $\bar{W}_{\mathrm{p}}$ can be converted to the more common clustering estimators used on large scales via the formalism presented in e.g. Hennawi et al. (2006a).

The projected real-space correlation function of quasars with a maximum velocity difference of $|\Delta v|<2000 \mathrm{~km} \mathrm{~s}^{-1}$ can be interpreted as

$w_{\mathrm{p}}(R, z)=\int_{-v_{\max } / H(z)}^{v_{\max } / H(z)} \xi_{\mathrm{s}}(R, s, z) \mathrm{d} s$,

where $v_{\max }=2000 \mathrm{~km} \mathrm{~s}^{-1}, H(z)$ is the expansion rate at redshift $z$ and $\xi_{\mathrm{s}}$ is the quasar correlation function in redshift space.

As discussed in Hennawi et al. (2006a), it is a good approximation to replace the redshift space correlation function $\xi_{\mathrm{s}}$ with its three-dimensional real-space counterpart $\xi(r)$. We measure the volume-averaged correlation function $\bar{W}_{\mathrm{p}}\left[R_{\min }, R_{\max }, z\right.$, abbreviated to $\left.\bar{W}_{\mathrm{p}}(z)\right]$ by integrating over the entire radial bin of proper distance $\left[R_{\min }, R_{\max }\right]$

$\bar{W}_{\mathrm{p}}(z)=\frac{\int_{-v_{\max } / H(z)}^{v_{\max } / H(z)} \int_{R_{\min }}^{R_{\max }} \xi(R, x, z) 2 \pi R \mathrm{~d} R \mathrm{~d} s}{V_{\text {shell }}}$,

where $\xi(R, x, z)$ is the correlation function and $V_{\text {shell }}$ is the volume of the cylindrical shell in redshift space over which we integrate

$V_{\text {shell }}=\pi\left(R_{\max }^{2}-R_{\min }^{2}\right)\left[\frac{2 v_{\max }}{H(z)}\right]$,

${ }^{10}$ e.g. http://classic.sdss.org/dr6/products/images/index.html. and then averaging the redshift-dependent $\bar{W}_{\mathrm{p}}$ in equation (5) over the redshift distribution of quasars in our sample.

We need to average $W_{\mathrm{p}}(z)$ over the redshift distribution of our sample in a given redshift bin in order to compare to our clustering measurement. To estimate the redshift distribution for our quasars of interest in any slice of redshift, we use the pure luminosity evolution (PLE) model of Croom et al. (2009) with $\alpha=-3.33$, $\beta=-1.42, \mathrm{M}^{\star}=-22.17$ and $\log \left(\phi^{\star}\right)=-5.84 \mathrm{Mpc}^{-3} \mathrm{mag}^{-1}$. We adopt this particular luminosity function as the sample of quasars studied in Croom et al. (2009) is a reasonable match $(0.4<z<2.6)$ to the redshift range of our sample and extends well beyond $(g<21.85)$ our magnitude limit.

Because we measure $\bar{W}_{\mathrm{p}}$ for quasars, the $\xi$ included in equation (5) is typically the correlation function of quasars, which we will denote $\xi_{\mathrm{Q}}$. We will adopt two typical theoretical forms for this function. First, a two-parameter power law of the form

$\xi_{\mathrm{Q}}(r)=\left(r / r_{0}\right)^{-\gamma}$,

where $r_{0}$ is the correlation length, defined as the most common (probable) separation between two quasars in the sample, and $\gamma$ is the exponent that best recreates the shape of quasar clustering. Secondly,

$\left.\xi_{\mathrm{Q}}(r)=A \xi_{(} r\right)$,

where $A$ is the ratio of the clustering amplitude of quasars to that of the underlying dark matter distribution and $\left.\xi_{(} r\right)$ is the correlation function of underlying dark matter, for which we adopt the model of Smith et al. (2003). In some places in Section 4, we use values of $r_{0}$ and $\gamma$, or a form for $\xi(r)$, that have been derived for the clustering of quasars or dark matter on Mpc scales. We then use equation (5) to project this Mpc-scale result down to our kpc scales of interest.

Phenomenologically, the formalism of equation (8) resembles that for the bias of tracers of dark matter (e.g. Kaiser 1984). We appreciate, though, that small-scale bias could change rapidly with scale and that the amplitude of quasar clustering is likely to be a complex function of several factors on non-linear scales. Any association we make between the parameter $A$ and the bias of dark matter $\left(b_{\mathrm{Q}}\right)$ in this work, therefore, is only to make comparisons between the amplitude of quasar clustering at $\mathrm{kpc}$ and Mpc scales. In essence, we adopt equation (8) only as an empirical parametrization of the amplitude of quasar clustering on kpc scales. We reserve models that have a more complex physical interpretation for a later paper.

\section{RESULTS AND DISCUSSION}

Our KDE-complete sample of confirmed binary quasars is $\sim 6$ times larger than any individual previous sample, allowing us to measure the scale dependence of quasar clustering at $\lesssim 40 h^{-1} \mathrm{kpc}$ with unparalleled precision. In addition, our large sample extends across multiple bins in redshift that each contain about as many binary quasars as any previous sample. This allows us to study the evolution of quasar clustering on these very small scales for the first time.

\subsection{The scale dependence of $\bar{W}_{\mathrm{p}}$ at $\lesssim 40 \mathrm{~h}^{-1} \mathrm{kpc}$}

We measure the volume-averaged projected correlation function $\left(\bar{W}_{\mathrm{p}}\right)$ of quasars in four bins of proper scale centred at 18.8, 22.8, 27.6 and $33.4 h^{-1} \mathrm{kpc}$, which contain 7, 14, 11 and 15 binary quasars, respectively. The bins were chosen to have the same width in logarithmic scale. The measured $\bar{W}_{\mathrm{p}}$ for each bin of proper separation together with the measured $\bar{W}_{\mathrm{p}}$ for the full sample at $\bar{z}=1.55$ 


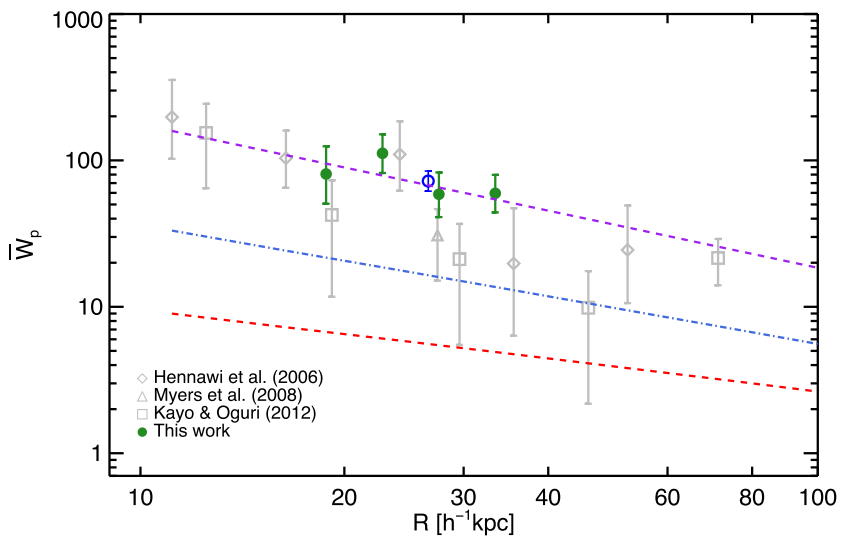

Figure 7. The projected correlation function of quasars in four bins of proper scale (filled green circles) as well as the measured correlation function for the full sample at $\bar{z}=1.55$ and $R=26.6 h^{-1} \mathrm{kpc}$ (open blue circle). The grey symbols depict similar measurements from previous studies. Where necessary, we have converted the comoving coordinates used in previous measurements to proper coordinates in order to compare with our data. The red and blue dashed and dot-dashed lines are the extrapolation of the $\bar{W}_{\mathrm{p}}$ correlation function reported on Mpc scales by Porciani et al. (2004) with $\left(\gamma=1.53, r_{0}=4.8 h^{-1} \mathrm{cMpc}\right)$ and $\left(\gamma=1.8, r_{0}=5.4 h^{-1} \mathrm{cMpc}\right)$, respectively. The purple line shows the best fit to the green data points, assuming a correlation length of $r_{0}=5.0 \mathrm{~h}^{-1} \mathrm{cMpc}$. This line has a powerlaw index of $\gamma=1.97 \pm 0.03$, indicating that the correlation function is steeper on kpc scales than has been estimated for many quasar samples on Mpc scales (as discussed further in Section 4.1)

Table 4. The volume-averaged correlation function for the four bins of proper separation displayed in Fig. 7. The last row corresponds to the full range of scales (the open blue circle in Fig. 7).

\begin{tabular}{lc}
\hline$R\left(h^{-1} \mathrm{kpc}\right)$ & $\bar{W}_{\mathrm{p}}$ \\
\hline 18.8 & $79.8_{-29.8}^{+43.5}$ \\
22.8 & $109.1_{-29.1}^{+38.0}$ \\
27.6 & $58.0_{-17.5}^{+23.7}$ \\
33.4 & $59.2_{-15.4}^{+19.9}$ \\
26.6 & $72.28_{-13.5}^{+15.2}$ \\
\hline
\end{tabular}

and $\bar{R}=26.6 h^{-1} \mathrm{kpc}$ is shown in Fig. 7. Multiple past works have argued that pair counts on small scales are independent and that clustering on these scales can be adequately described by a Poisson distribution (e.g. Shanks \& Boyle 1994; Croom \& Shanks 1996; Myers et al. 2006; Chatterjee et al. 2012, 2013). We therefore adopt Poisson errors from Gehrels (1986) for our measurements of $\bar{W}_{\mathrm{p}}$.

Table 4 lists our measured $\bar{W}_{\mathrm{p}}$ in each bin of proper separation and for our full KDE-complete sample of 47 binary quasars.

Fig. 7 also compares our measurement of $\bar{W}_{\mathrm{p}}$ to previous estimates of quasar clustering on small scales at redshifts of $0.5 \lesssim z$ $\lesssim 2.5$. Hennawi et al. (2006a) constructed a large homogeneous catalogue of binary quasars from SDSS DR3 and used a subsample of them to measure quasar clustering on small scales. The clustering subsample of Hennawi et al. (2006a) included 23 binary quasars on proper scales of $10 \lesssim R \lesssim 100 \mathrm{~h}^{-1} \mathrm{kpc}$. Myers et al. (2008) built on this work by discovering 10 new binary quasars in the SDSS DR4 KDE catalogue and used them to study quasar clustering on a specific range of very small proper scales $\left(20 \lesssim R \lesssim 30 h^{-1} \mathrm{kpc}\right)$. More recently, Kayo \& Oguri (2012) compiled a sample of binary quasars from observations conducted across SDSS DR7 as part of the SDSS Quasar Lens Search (e.g. Inada et al. 2012) and used 26 binaries with comoving separations of $10-200 h^{-1}$ ckpc (proper scales of $5 \lesssim R \lesssim 100 h^{-1} \mathrm{kpc}$ ) to measure $\bar{W}_{\mathrm{p}}$. The sample of Kayo \& Oguri (2012) only shares four binaries with that of Hennawi et al. (2006a) and a further two with Myers et al. (2008). This is largely because the sample of Kayo \& Oguri (2012) is more complete than the sample of Hennawi et al. (2006a), covers a larger range of scales than the sample of Myers et al. (2008) and covers a larger portion of the SDSS footprint as compared to both studies.

The sample of Kayo \& Oguri (2012) contains only four binary quasars on scales of $17 \lesssim R \lesssim 36 h^{-1} \mathrm{kpc}$, and two of these are the pairs that Kayo \& Oguri (2012) incorporated from Myers et al. (2008). Further, the clustering subsample of Hennawi et al. (2006a) includes only eight binary quasars on proper scales of $17 \lesssim R \lesssim$ $36 h^{-1} \mathrm{kpc}$. Our KDE-complete sample of binary quasars is thus $\sim 6 \times$ larger than any previous statistically homogeneous sample at $R \sim 25 h^{-1} \mathrm{kpc}$ and so can substantially improve the accuracy of quasar clustering measurements on small scales. Table 5 contains the full list of 230 candidate quasar pairs drawn from our parent sample, together with available spectroscopic confirmations and redshifts from our own and previous campaigns. The blue open circle in Fig. 7 shows the statistical significance of our measurement compared to recent such measurements on kpc scales. Our KDEcomplete sample is about $4 \times$ larger than all other combined samples at $17 \lesssim R \lesssim 36 h^{-1} \mathrm{kpc}$ (Table 6 lists the sample of 47 binary quasars that define our KDE-complete sample). This essentially means that our results can be used to improve constraints on kpc-scale quasar clustering by a factor of 2 compared to previous work.

The real-space correlation function of quasars can be modelled by a simple power law (see equation 7). Quasars in our redshift range of interest $(0.4<z<2.3)$ have been argued to have a range of power-law indexes based on clustering measurements conducted on Mpc scales. The sample that best matches our luminosity and redshift range $\left(b_{\mathrm{J}}<20.85 ; 0.3<z<2.2\right)$ is that of the $2 \mathrm{dF}$ QSO Redshift Survey (2QZ; Croom et al. 2004). For the 2QZ, Porciani et al. (2004) measured a best-fitting power law of $\gamma=1.53$ for $r_{0}=4.8 h^{-1} \mathrm{cMpc}$, rising to $\gamma=1.8$ for $r_{0}=5.4 h^{-1} \mathrm{cMpc}$. Ross et al. (2009) found that the clustering of brighter quasars from the SDSS $(i<19.1 ; 0.3<z<2.2)$ required a steeper power law of $\gamma=1.90_{-0.03}^{+0.04}$ for $r_{0}=5.5 \mathrm{~h}^{-1} \mathrm{cMpc}$. The red dashed and blue dotdashed lines in Fig. 7 compare the best-fitting power laws from Porciani et al. (2004) to our results, and it is clear that our data necessitate a much steeper power law. Fixing the correlation length to $r_{0}=5 \mathrm{~h}^{-1} \mathrm{cMpc}$, we use a maximum likelihood fitting procedure to determine that our data require a power-law index of $\gamma=1.97$ \pm 0.03 , which is plotted as the purple dashed line in Fig. 7. This power-law index is far in excess of the results of Porciani et al. (2004) but is in reasonable agreement with the Mpc-scale clustering of substantially brighter SDSS quasars from Ross et al. (2009). Our results also support the study of Kayo \& Oguri (2012), who found a power law of $\gamma=1.92 \pm 0.04$ for $r_{0}=5.4 h^{-1} \mathrm{cMpc}$ from a study of the clustering of bright $(i<19.1 ; 0.6<z<2.2)$ SDSS quasars on proper scales of $4 \lesssim R \lesssim 85 h^{-1} \mathrm{kpc}$.

At low redshift ( $z \sim 0.5$ and below), quasars appear to be roughly unbiased (e.g. Croom et al. 2005) and cluster similarly to $L^{*}$ galaxies. Given that quasars are thought to be merger-driven (e.g. Hopkins et al. 2006, 2007), it is interesting to compare the overall shape of the correlation function of galaxies and of quasars at similar redshift. Any excess in quasar clustering compared to galaxies might indicate that quasars ignite in particularly grouped or 'merger-prone' environments (again see Hopkins et al. 2007). For 
Table 5. Candidate quasar pairs drawn from our parent sample. ${ }^{a}$

\begin{tabular}{|c|c|c|c|c|c|c|c|c|}
\hline $\begin{array}{l}\Delta \theta \\
(\operatorname{arcsec})\end{array}$ & Obs. stat. & $\begin{array}{c}\alpha \\
(\mathrm{J} 2000)\end{array}$ & $\begin{array}{c}\delta \\
(\mathrm{J} 2000)\end{array}$ & $i$ & $g$ & Classification & $z_{\mathrm{spec}}$ & QQ? \\
\hline \multirow[t]{2}{*}{1.449} & 2 & 184.19140 & 35.49488 & 19.88 & 20.40 & Q & -1 & 4 \\
\hline & 2 & 184.19190 & 35.49486 & 19.08 & 19.39 & Q & 2.013 & \\
\hline \multirow[t]{2}{*}{1.693} & 2 & 204.77974 & 13.17768 & 18.91 & 19.03 & Q & 2.241 & 4 \\
\hline & 2 & 204.78015 & 13.17741 & 18.87 & 18.96 & Q & 2.237 & \\
\hline \multirow[t]{2}{*}{1.762} & 0 & 19.55013 & -1.07848 & 19.99 & 21.00 & $\mathrm{U}$ & 0.740 & 1 \\
\hline & 0 & 19.55053 & -1.07820 & 20.35 & 20.40 & $\mathrm{U}$ & -1 & \\
\hline \multirow[t]{2}{*}{1.897} & 0 & 177.82866 & 46.87642 & 20.28 & 20.68 & $\mathrm{U}$ & -1 & 1 \\
\hline & 0 & 177.82939 & 46.87626 & 19.04 & 20.41 & $\mathrm{U}$ & -1 & \\
\hline \multirow[t]{2}{*}{1.939} & 0 & 230.20850 & 26.62804 & 19.07 & 19.35 & $\mathrm{Q}$ & -1 & 1 \\
\hline & 2 & 230.20911 & 26.62802 & 19.00 & 19.21 & Q & 1.365 & \\
\hline \multirow[t]{2}{*}{1.999} & 2 & 228.91032 & 15.19300 & 18.37 & 18.70 & Q & 2.052 & 4 \\
\hline & 2 & 228.91080 & 15.19331 & 18.05 & 18.16 & $\mathrm{Q}$ & 2.054 & \\
\hline \multirow[t]{2}{*}{2.102} & 0 & 112.11562 & 26.11704 & 19.65 & 19.98 & $\mathrm{U}$ & -1 & 1 \\
\hline & 0 & 112.11615 & 26.11737 & 18.84 & 18.90 & $\mathrm{U}$ & -1 & \\
\hline \multirow[t]{2}{*}{2.173} & 0 & 146.32053 & 22.41586 & 20.79 & 20.94 & $\mathrm{U}$ & -1 & 1 \\
\hline & 0 & 146.32063 & 22.41646 & 20.72 & 20.81 & $\mathrm{U}$ & -1 & \\
\hline \multirow[t]{2}{*}{2.196} & 0 & 250.07547 & 10.75175 & 19.59 & 20.42 & $\mathrm{U}$ & -1 & 1 \\
\hline & 0 & 250.07599 & 10.75141 & 17.83 & 18.39 & $\mathrm{U}$ & -1 & \\
\hline \multirow[t]{2}{*}{2.267} & 0 & 244.24273 & 36.50716 & 20.42 & 20.17 & $\mathrm{U}$ & -1 & 1 \\
\hline & 0 & 244.24332 & 36.50758 & 20.40 & 20.12 & $\mathrm{U}$ & -1 & \\
\hline \multirow[t]{2}{*}{2.316} & 2 & 250.79727 & 31.93844 & 19.53 & 20.00 & Q & 0.587 & 1 \\
\hline & 0 & 250.79745 & 31.93907 & 19.47 & 19.89 & $\mathrm{U}$ & -1 & \\
\hline \multirow[t]{2}{*}{2.453} & 2 & 145.64575 & 23.17533 & 19.76 & 19.91 & $\mathrm{Q}$ & 1.833 & 3 \\
\hline & 2 & 145.64598 & 23.17468 & 19.70 & 19.80 & Q & 1.833 & \\
\hline \multirow[t]{2}{*}{2.654} & 2 & 158.83012 & 7.88232 & 20.16 & 20.62 & $\mathrm{Q}$ & 1.218 & 3 \\
\hline & 2 & 158.83069 & 7.88278 & 19.02 & 19.10 & Q & 1.215 & \\
\hline \multirow[t]{2}{*}{2.678} & 0 & 161.08777 & 4.49745 & 20.60 & 20.99 & $\mathrm{U}$ & -1 & 1 \\
\hline & 0 & 161.08844 & 4.49713 & 19.35 & 19.73 & $\mathrm{U}$ & -1 & \\
\hline \multirow[t]{2}{*}{2.695} & 0 & 115.60587 & 24.86230 & 20.64 & 20.90 & $\mathrm{U}$ & -1 & 1 \\
\hline & 0 & 115.60665 & 24.86254 & 20.56 & 20.74 & $\mathrm{U}$ & -1 & \\
\hline \multirow[t]{2}{*}{2.829} & 1 & 182.49029 & 11.61649 & 20.46 & 20.76 & Q & 0.899 & 3 \\
\hline & 1 & 182.49049 & 11.61573 & 20.40 & 20.65 & Q & 0.904 & \\
\hline \multirow[t]{2}{*}{2.868} & 0 & 322.49351 & 12.00661 & 20.71 & 20.88 & $\mathrm{~S}$ & -1 & 1 \\
\hline & 0 & 322.49390 & 12.00731 & 20.50 & 20.49 & $\mathrm{U}$ & -1 & \\
\hline \multirow[t]{2}{*}{2.903} & 2 & 227.17583 & 33.46739 & 20.60 & 20.44 & Q & 0.877 & 3 \\
\hline & 1 & 227.17590 & 33.46820 & 20.56 & 20.38 & $\mathrm{Q}$ & 0.878 & \\
\hline \multirow[t]{2}{*}{2.912} & 1 & 152.07303 & 17.25558 & 20.27 & 20.67 & Q & 1.087 & 3 \\
\hline & 1 & 152.07367 & 17.25506 & 20.19 & 20.52 & Q & 1.083 & \\
\hline \multirow[t]{2}{*}{2.918} & 1 & 143.15840 & 29.40301 & 20.94 & 20.66 & $\mathrm{U}$ & -1 & 1 \\
\hline & 0 & 143.15854 & 29.40221 & 20.90 & 20.59 & $\mathrm{U}$ & -1 & \\
\hline \multirow[t]{2}{*}{2.925} & 2 & 150.36812 & 50.46623 & 17.71 & 18.34 & Q & 1.845 & 4 \\
\hline & 2 & 150.36922 & 50.46581 & 17.32 & 17.55 & $\mathrm{Q}$ & 1.841 & \\
\hline 2.933 & 1 & 158.41804 & 2.47517 & 19.65 & 20.28 & $\mathrm{U}$ & -1 & 1 \\
\hline & 1 & 158.41884 & 2.47531 & 19.91 & 20.14 & Q & 1.833 & \\
\hline 2.993 & 2 & 207.37436 & 12.45193 & 18.73 & 19.32 & $\mathrm{Q}$ & 1.722 & 4 \\
\hline & 1 & 207.37503 & 12.45245 & 18.66 & 19.19 & $\mathrm{Q}$ & 1.722 & \\
\hline
\end{tabular}

(1) Angular separation of the two members of the pair; (2) the observational status of the pair is ' 0 ' if there is insufficient information to determine the redshift of a candidate, ' 1 ' for sources confirmed by this study and ' 2 ' for sources confirmed in previous studies (Schneider et al. 2005; Hennawi et al. 2006a; Inada et al. 2008; Myers et al. 2008; Oguri et al. 2008, 2012; Schneider et al. 2010; Prochaska et al. 2013, 2014; Pâris et al. 2016); (3-4) source coordinates in degrees; (5-6) dereddened $i$ and $g$ magnitudes; (7) spectroscopic classification: Q=Quasar G=Galaxy, $\mathrm{S}=\mathrm{Star}, \mathrm{U}=\mathrm{No}$ Spectrum, $\mathrm{NQ}=\mathrm{A}$ spectrum exists but it did not yield a definitive classification (i.e. 'Not a quasar'); (8) the measured or reported spectroscopic redshift for the members, -1 for objects with no redshift; (9) classification of the pair as (i) lacking sufficient spectroscopic information to define its nature, (ii) a projected pair (star-star, star-quasar, two quasars at different redshifts etc.), (iii) a binary quasar, (iv) a gravitational lens.

${ }^{a}$ The full table is available in the electronic version of this paper.

example, Watson et al. (2010) suggest that enhanced quasar activity by mergers might be responsible for the shape differences between the correlation function of luminous red galaxies (LRGs) and quasars on very small scales. Large spectroscopic galaxy surveys are now approaching $z \sim 1$, so it is becoming realistic to compare quasar and galaxy correlation functions in similar redshift ranges.
Recent galaxy clustering results on $\mathrm{Mpc}$ scales tend to find powerlaw slopes that are shallower than $\gamma=2$. For example, Favole et al. (2016) find $\gamma=1.6 \pm 0.1$ in redshift space for $s_{0}=(5.3$ $\pm 0.2) h^{-1} \mathrm{cMpc}$ for emission line galaxies at $z \sim 0.8$. Coil et al. (2016) find a range of power-law slopes for blue and red galaxies from the PRIMUS survey over the redshift range $0.4 \lesssim z \lesssim 0.9$. For populations that have $r_{0}$ consistent (within their $1 \sigma$ errors) 
Table 6. Complete sample of 47 spectroscopically confirmed binaries.

\begin{tabular}{|c|c|c|c|c|c|c|c|c|}
\hline Name & $\begin{array}{c}\text { RA } \\
(\mathrm{J} 2000)\end{array}$ & $\begin{array}{c}\text { Dec. } \\
(\mathrm{J} 2000)\end{array}$ & $g$ & $i$ & $\begin{array}{c}\Delta \theta \\
\operatorname{arcsec}\end{array}$ & $z_{\text {spec }}$ & $\begin{array}{c}|\Delta v| \\
\left(\mathrm{km} \mathrm{s}^{-1}\right)\end{array}$ & $\begin{array}{l}R \\
\left(h^{-1} \mathrm{kpc}\right)\end{array}$ \\
\hline SDSS J0718+4020 A & 109.51462 & 40.35075 & 20.04 & 20.16 & \multirow[t]{2}{*}{5.926} & \multirow[t]{2}{*}{1.838} & \multirow[t]{2}{*}{0} & \multirow[t]{2}{*}{34.6} \\
\hline SDSS J0718+4020 B & 109.51288 & 40.34978 & 20.66 & 21.07 & & & & \\
\hline SDSS J0751+1303 A & 117.76192 & 13.06113 & 20.31 & 20.82 & \multirow[t]{2}{*}{6.166} & \multirow[t]{2}{*}{1.545} & \multirow[t]{2}{*}{1300} & \multirow[t]{2}{*}{36.1} \\
\hline SDSS J0751+1303 B & 117.76159 & 13.05944 & 20.38 & 20.94 & & & & \\
\hline SDSS J0813+5416 A & 123.30461 & 54.27972 & 17.27 & 17.24 & \multirow[t]{2}{*}{5.042} & \multirow[t]{2}{*}{0.778} & \multirow[t]{2}{*}{200} & \multirow[t]{2}{*}{26.1} \\
\hline SDSS J0813+5416 B & 123.30266 & 54.28054 & 20.20 & 20.25 & & & & \\
\hline SDSS J0818+3623 A & 124.63308 & 36.38616 & 17.73 & 17.99 & \multirow[t]{2}{*}{6.094} & \multirow[t]{2}{*}{1.961} & \multirow[t]{2}{*}{0} & \multirow[t]{2}{*}{35.3} \\
\hline SDSS J0818+3623 B & 124.63222 & 36.38770 & 19.26 & 19.75 & & & & \\
\hline SDSS J0846+2709 A & 131.60213 & 27.16733 & 20.44 & 20.43 & \multirow[t]{2}{*}{4.637} & \multirow[t]{2}{*}{2.195} & 0 & 26.5 \\
\hline SDSS J0846+2709 B & 131.60140 & 27.16622 & 20.46 & 20.66 & & & & \\
\hline SDSS J0916+3252 A & 139.24397 & 32.87321 & 19.38 & 19.73 & 6.122 & 1.911 & 600 & 35.6 \\
\hline SDSS J0916+3252 B & 139.24195 & 32.87304 & 19.75 & 20.10 & & & & \\
\hline SDSS J0922-0117 A & 140.57307 & -1.29715 & 18.64 & 18.93 & 6.032 & 1.677 & 1400 & 35.3 \\
\hline SDSS J0922-0117 B & 140.57305 & -1.29883 & 19.48 & 19.83 & & & & \\
\hline SDSS J0954+1920 A & 148.62408 & 19.33632 & 18.41 & 18.58 & 4.376 & 1.744 & 0 & 25.6 \\
\hline SDSS J0954+1920 B & 148.62282 & 19.33660 & 19.96 & 20.29 & & & & \\
\hline SDSS J0959+5449 A & 149.78113 & 54.81844 & 19.74 & 20.04 & 3.945 & 1.956 & 200 & 22.9 \\
\hline SDSS J0959+5449 B & 149.77942 & 54.81892 & 20.29 & 20.61 & & & & \\
\hline SDSS J1048+0950 A & 162.19373 & 9.83695 & 20.56 & 20.74 & 4.447 & 1.666 & 0 & 26.1 \\
\hline SDSS J1048+0950 B & 162.19254 & 9.83652 & 20.61 & 20.84 & & & & \\
\hline SDSS J1145+2857 A & 176.26947 & 28.95353 & 19.97 & 20.24 & 4.085 & 2.173 & 100 & 23.4 \\
\hline SDSS J1145+2857 B & 176.26818 & 28.95363 & 20.55 & 20.63 & & & & \\
\hline SDSS J1147+3305 A & 176.93478 & 33.08547 & 17.47 & 17.58 & 4.691 & 1.164 & 1000 & 26.9 \\
\hline SDSS J1147+3305 B & 176.93325 & 33.08565 & 20.14 & 20.18 & & & & \\
\hline SDSS J1158+1355 A & 179.71272 & 13.92666 & 20.78 & 20.66 & 3.237 & 2.062 & 1800 & 18.7 \\
\hline SDSS J1158+1355 B & 179.71198 & 13.92718 & 20.85 & 20.78 & & & & \\
\hline SDSS J1207+1408 A & 181.86359 & 14.13900 & 19.97 & 20.11 & 3.949 & 1.795 & 500 & 23.1 \\
\hline SDSS J1207+1408 B & 181.86292 & 14.13811 & 20.03 & 20.37 & & & & \\
\hline SDSS J1215+0225 A & 183.94466 & 2.43279 & 19.51 & 19.69 & 5.729 & 1.445 & 0 & 33.5 \\
\hline SDSS J1215+0225 B & 183.94425 & 2.43125 & 19.55 & 19.77 & & & & \\
\hline SDSS J1219+2541 A & 184.89709 & 25.68951 & 19.61 & 19.87 & 5.897 & 1.596 & 200 & 34.6 \\
\hline SDSS J1219+2541 B & 184.89556 & 25.68862 & 18.64 & 20.07 & & & & \\
\hline SDSS J1235+0434 A & 188.98030 & 68.60752 & 19.51 & 19.64 & 3.513 & 1.529 & 1800 & 20.6 \\
\hline SDSS J1235+0434 B & 188.97826 & 68.60689 & 19.54 & 19.72 & & & & \\
\hline SDSS J1259+1241 A & 194.98174 & 12.69828 & 19.73 & 19.99 & 3.554 & 2.180 & 900 & 20.3 \\
\hline SDSS J1259+1241 B & 194.98110 & 12.69752 & 19.78 & 20.09 & & & & \\
\hline SDSS J1303+5100 A & 195.85907 & 51.01311 & 19.98 & 20.33 & 3.806 & 1.686 & 200 & 22.3 \\
\hline SDSS J1303+5100 B & 195.85893 & 51.01417 & 20.34 & 20.54 & & & & \\
\hline SDSS J1320+3056 A & 200.09435 & 30.93842 & 19.65 & 19.90 & 4.745 & 1.597 & 500 & 27.8 \\
\hline SDSS J1320+3056 B & 200.09394 & 30.93969 & 19.68 & 19.94 & & & & \\
\hline SDSS J1337+6012 A & 204.30472 & 60.20183 & 18.34 & 18.55 & 3.118 & 1.721 & 1500 & 18.2 \\
\hline SDSS J1337+6012 B & 204.30452 & 60.20269 & 19.70 & 20.05 & & & & \\
\hline SDSS J1339+6208 A & 204.75824 & 62.14766 & 19.96 & 20.26 & 3.89 & 1.799 & 1800 & 22.7 \\
\hline SDSS J1339+6208 B & 204.75796 & 62.14659 & 20.36 & 20.90 & & & & \\
\hline SDSS J1344+1948 A & 206.12888 & 19.81089 & 19.95 & 20.06 & 4.694 & 1.534 & 200 & 27.5 \\
\hline SDSS J1344+1948 B & 206.12883 & 19.80959 & 20.25 & 20.48 & & & & \\
\hline SDSS J1418+2441 A & 214.73140 & 24.68464 & 19.83 & 20.15 & 4.504 & 0.573 & 400 & 20.5 \\
\hline SDSS J1418+2441 B & 214.73091 & 24.68581 & 19.87 & 20.23 & & & & \\
\hline SDSS J1426+0719 A & 216.51802 & 7.32501 & 19.82 & 20.03 & 4.271 & 1.324 & 300 & 24.8 \\
\hline SDSS J1426+0719 B & 216.51778 & 7.32385 & 20.59 & 20.82 & & & & \\
\hline SDSS J1430+0714 A & 217.51202 & 7.23648 & 19.02 & 19.39 & 5.414 & 1.245 & 1700 & 31.3 \\
\hline SDSS J1430+0714 B & 217.51110 & 7.23767 & 19.74 & 20.27 & & & & \\
\hline
\end{tabular}


Table 6 - continued

\begin{tabular}{|c|c|c|c|c|c|c|c|c|}
\hline Name & $\begin{array}{c}\text { RA } \\
(\mathrm{J} 2000)\end{array}$ & $\begin{array}{l}\text { Dec. } \\
\text { (J2000) }\end{array}$ & $g$ & $i$ & $\begin{array}{c}\Delta \theta \\
(\operatorname{arcsec})\end{array}$ & $z_{\text {spec }}$ & $\begin{array}{c}|\Delta v| \\
\left(\mathrm{km} \mathrm{s}^{-1}\right)\end{array}$ & $\begin{array}{l}R \\
\left(h^{-1} \mathrm{kpc}\right)\end{array}$ \\
\hline SDSS J1430+1539 A & 217.51620 & 15.66371 & 19.76 & 19.64 & \multirow[t]{2}{*}{6.265} & \multirow[t]{2}{*}{0.912} & \multirow[t]{2}{*}{200} & \multirow[t]{2}{*}{34.0} \\
\hline SDSS J1430+1539 B & 217.51486 & 15.66256 & 19.75 & 20.06 & & & & \\
\hline SDSS J1431+2705 A & 217.77074 & 27.09129 & 20.10 & 20.19 & \multirow[t]{2}{*}{5.913} & \multirow[t]{2}{*}{2.261} & \multirow[t]{2}{*}{900} & \multirow[t]{2}{*}{33.6} \\
\hline SDSS J1431+2705 B & 217.76937 & 27.09018 & 20.13 & 20.25 & & & & \\
\hline SDSS J1433+1450 A & 218.46286 & 14.83489 & 19.21 & 19.38 & \multirow[t]{2}{*}{3.336} & \multirow[t]{2}{*}{1.506} & \multirow[t]{2}{*}{500} & \multirow[t]{2}{*}{19.5} \\
\hline SDSS J1433+1450 B & 218.46227 & 14.83561 & 19.25 & 19.45 & & & & \\
\hline SDSS J1439+0601 A & 219.95763 & 6.01756 & 20.39 & 20.80 & \multirow[t]{2}{*}{5.329} & \multirow[t]{2}{*}{1.151} & \multirow[t]{2}{*}{0} & \multirow[t]{2}{*}{30.4} \\
\hline SDSS J1439+0601 B & 219.95697 & 6.01889 & 20.47 & 20.94 & & & & \\
\hline SDSS J1440+1515 A & 220.24983 & 15.26339 & 19.55 & 19.97 & \multirow[t]{2}{*}{3.852} & \multirow[t]{2}{*}{1.153} & \multirow[t]{2}{*}{0} & \multirow[t]{2}{*}{22.0} \\
\hline SDSS J1440+1515 B & 220.24968 & 15.26233 & 20.48 & 20.65 & & & & \\
\hline SDSS J1444+5413 A & 221.09413 & 54.22240 & 20.05 & 20.25 & \multirow[t]{2}{*}{3.446} & \multirow[t]{2}{*}{1.584} & \multirow[t]{2}{*}{0} & 20.2 \\
\hline SDSS J1444+5413 B & 221.09255 & 54.22263 & 20.15 & 20.83 & & & & \\
\hline SDSS J1457+2516 A & 224.49562 & 25.28052 & 19.71 & 19.82 & 5.689 & 1.376 & 0 & 33.2 \\
\hline SDSS J1457+2516 B & 224.49422 & 25.27957 & 19.82 & 19.99 & & & & \\
\hline SDSS J1458+5448 A & 224.61137 & 54.80367 & 19.62 & 20.49 & 5.142 & 1.905 & 300 & 29.9 \\
\hline SDSS J1458+5448 B & 224.60902 & 54.80413 & 20.47 & 20.80 & & & & \\
\hline SDSS J1507+2903 A & 226.94681 & 29.05924 & 19.86 & 19.88 & 4.349 & 0.863 & 0 & 23.2 \\
\hline SDSS J1507+2903 B & 226.94545 & 29.05949 & 20.19 & 20.44 & & & & \\
\hline SDSS J1512+2951 A & 228.24347 & 29.86401 & 18.38 & 18.58 & 5.312 & 1.809 & 500 & 31.0 \\
\hline SDSS J1512+2951 B & 228.24194 & 29.86337 & 19.52 & 20.83 & & & & \\
\hline SDSS J1518+2959 A & 229.59763 & 29.99099 & 19.87 & 20.17 & 5.281 & 1.249 & 800 & 30.5 \\
\hline SDSS J1518+2959 B & 229.59607 & 29.99042 & 19.92 & 20.25 & & & & \\
\hline SDSS J1530+5304 A & 232.66176 & 53.06685 & 20.28 & 20.64 & 4.114 & 1.535 & 200 & 24.1 \\
\hline SDSS J1530+5304 B & 232.66068 & 53.06779 & 20.31 & 20.70 & & & & \\
\hline SDSS J1545+2755 A & 236.31659 & 27.93363 & 19.43 & 19.70 & 3.735 & 1.494 & 100 & 21.9 \\
\hline SDSS J1545+2755 B & 236.31556 & 27.93314 & 20.24 & 20.66 & & & & \\
\hline SDSS J1553+2230 A & 238.37730 & 22.50399 & 20.50 & 20.70 & 6.111 & 0.641 & 1300 & 29.2 \\
\hline SDSS J1553+2230 B & 238.37596 & 22.50284 & 20.63 & 20.93 & & & & \\
\hline SDSS J1559+2640 A & 239.78497 & 26.67552 & 19.66 & 19.81 & 5.367 & 0.870 & 1000 & 28.7 \\
\hline SDSS J1559+2640 B & 239.78424 & 26.67686 & 20.29 & 20.44 & & & & \\
\hline SDSS J1602+1314 A & 240.61542 & 13.23796 & 19.93 & 20.08 & 5.324 & 2.018 & 100 & 30.8 \\
\hline SDSS J1602+1314 B & 240.61448 & 13.23680 & 20.35 & 20.46 & & & & \\
\hline SDSS J1606+2900 A & 241.51259 & 29.01413 & 18.37 & 18.29 & 3.446 & 0.770 & 300 & 17.7 \\
\hline SDSS J1606+2900 B & 241.51172 & 29.01355 & 18.36 & 18.50 & & & & \\
\hline SDSS J1635+2911 A & 248.79294 & 29.18783 & 20.11 & 20.33 & 4.917 & 1.587 & 0 & 28.8 \\
\hline SDSS J1635+2911 B & 248.79228 & 29.18907 & 20.16 & 20.42 & & & & \\
\hline SDSS J1637+2636 A & 249.25389 & 26.60274 & 18.97 & 19.11 & 3.904 & 1.961 & 0 & 22.6 \\
\hline SDSS J1637+2636 B & 249.25367 & 26.60381 & 20.52 & 20.60 & & & & \\
\hline SDSS J1649+1733 A & 252.37083 & 17.55239 & 19.23 & 19.45 & 3.618 & 2.080 & 0 & 20.8 \\
\hline SDSS J1649+1733 B & 252.36997 & 17.55182 & 19.42 & 19.77 & & & & \\
\hline SDSS J1723+5904 A & 260.82260 & 59.07956 & 18.56 & 18.78 & 3.721 & 1.597 & 400 & 21.8 \\
\hline SDSS J1723+5904 B & 260.82211 & 59.07855 & 20.07 & 20.32 & & & & \\
\hline
\end{tabular}

Columns: (1) name of the members of the binary, where the brighter and fainter quasars in the pair in $g$ band are referred to as 'A' or 'B', respectively; (2-3) right ascension and declination of each quasar; (4-5) $g$ and $i$ magnitude of each quasar; (6) angular separation of the quasars in the binary; (7) spectroscopic redshift for the binary; (8) the velocity difference between the quasars in the binary; (9) the transverse proper separation between the quasars in the binary.

with $5 h^{-1} \mathrm{cMpc}$, Coil et al. (2016) find $\gamma=1.6-1.7$ with an error less than \pm 0.1 . On smaller scales, galaxy clustering may steepen, however. Masjedi et al. (2006) tracked the clustering of $z \sim 0.25$ SDSS LRGs down to scales of $\sim 10 \mathrm{~h}^{-1} \mathrm{kpc}$ and estimated $\gamma \sim 2.0$, although they also found a large correlation length of $r_{0} \sim 10 h^{-1} \mathrm{cMpc}$. Zehavi et al. (2011) found power-law slopes ranging from $\gamma \sim 1.8$ to 2.0 for $r_{0} \sim 4.5-10.4 h^{-1} \mathrm{cMpc}$ down to comoving distances of $\sim 100 h^{-1}$ ckpc for SDSS galaxies at $z \lesssim$ 0.25 . For samples that have $r_{0}$ in the range $4.5-5.5 h^{-1} \mathrm{cMpc}$, Zehavi et al. (2011) find $\gamma \sim 1.8-1.9$. More recently and at higher redshift, Zhai et al. (2016) find $\gamma \sim 1.95$ down to scales of $\sim 300 \mathrm{~h}^{-1} \mathrm{ckpc}$ for the clustering of $z \sim 0.7$ LRGs drawn from the SDSS-IV/extended Baryon Oscillation Spectroscopic Survey. Our inferred power law of $\gamma=1.97 \pm 0.03$ for $r_{0}=5 h^{-1} \mathrm{cMpc}$ is therefore at the steeper 

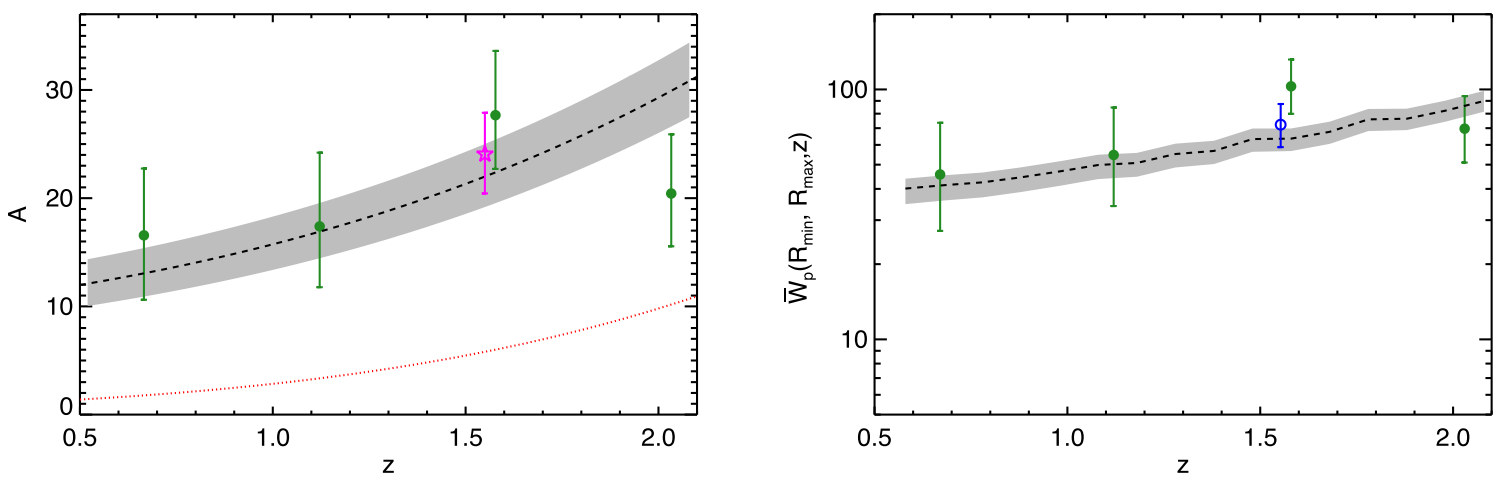

Figure 8. Left: our deduced quasar clustering amplitude at $R \sim 25 h^{-1} \mathrm{kpc}$ in each bin of redshift. The pink star depicts the amplitude derived from our measurement of quasar clustering for our full sample $(A=24.1 \pm 3.6)$. The red dotted line is the model for the evolution of quasar bias on Mpc scales proposed by Croom et al. (2005); $b_{\mathrm{Q}}^{2}(z)=\left(0.53+0.289(1+z)^{2}\right)^{2}$. The black dashed line depicts the best-fitting value we find for a one-parameter fit of $A(z)=\left(c+0.289(1+z)^{2}\right)^{2}$, which is $c=2.81 \pm 0.31$. The grey envelope depicts the $1 \sigma$ confidence interval for the fitted parameter $c$. Right: the projected correlation function of quasars in four bins of redshift. Each redshift bin spans the full range of proper scales of our KDE-complete sample $(17.0 \leq R \leq$ $36.2 h^{-1} \mathrm{kpc}$ ). The black dashed line is the calculated $\bar{W}_{\mathrm{p}}$ for the full redshift range of our KDE-complete sample, using the model from the left-hand panel. The grey envelope is the translation of the confidence intervals from the left-hand panel based on the relationship between $A$ and $\bar{W}_{\mathrm{p}}$ outlined in Section 3.2 .

end of what has been measured for galaxies, but is not inconsistent with measurements at higher redshift that sample smaller scales. A detailed theoretical analysis, such as the halo occupation distribution (HOD; Berlind \& Weinberg 2002) formalism, should be able to use our measurements to better quantify whether quasar clustering exceeds galaxy clustering on kpc scales, or whether quasars occupy similar haloes to certain types of galaxies. We defer such a detailed HOD analysis to a later paper.

\subsection{Redshift dependence of $\bar{W}_{\mathrm{p}}$}

Measurements of the evolution of quasar clustering on Mpc scales (e.g. Croom et al. 2005), in combination with the quasar luminosity function, have helped to constrain fuelling models for quasars and provided a framework to link quasar activity to galaxy formation (see, e.g. Hopkins et al. 2007, and references therein). Broadly, the quasar correlation length on Mpc scales does not appear to evolve by more than a factor of $\sim 2$ over the range $0.5 \lesssim z \lesssim 2.5$ (see, e.g. Eftekharzadeh et al. 2015, and references therein). This, in turn, implies that quasar bias increases significantly between redshift 0.5 and 2.5 and that the characteristic mass of the dark matter haloes that host quasars is roughly constant across this redshift range. Myers et al. (2007b) estimated how quasar clustering on small scales changes with redshift using a sample of 91 photometrically classified candidate quasars and found that UV-excess quasars at $28 h^{-1} \mathrm{kpc}$ cluster $>5$ times $(\sim 2 \sigma)$ higher at $z>2$ than at $z<2$. However, the evolution of quasar clustering on proper scales of $<50 h^{-1} \mathrm{kpc}$ has not yet been measured using a spectroscopically confirmed sample of quasar pairs, likely because sample sizes have never been sufficiently large to bin by redshift. With the unprecedentedly large number of binary quasars in, and wide redshift range of, our KDEcomplete sample, we can make this measurement for the first time.

We divide our KDE-complete sample of quasar pairs into four bins of redshift of similar width $(\Delta z \simeq 0.46)$ centred at $z=0.67$, $1.12,1.58$ and 2.03 . These bins contain $6,7,20$ and 14 quasar pairs, respectively. ${ }^{11}$ We then measure the correlation function $\bar{W}_{\mathrm{p}}\left(R_{\min }, R_{\max }\right)$ in each bin of redshift over the full range of proper

\footnotetext{
${ }^{11}$ Choosing the redshift slices such that they contain the same number of pairs would cause some bins to be very narrow.
}

scales of our sample $\left(17.0<R<36.2 h^{-1} \mathrm{kpc}\right)$. We plot the results of this analysis in the right-hand panel of Fig. 8. Having measured the volume-averaged correlation function in four slices of redshift, we use the method described in Section 3.2 to derive the amplitude of quasar clustering (A from equation 8) in each bin of redshift. The left-hand panel of Fig. 8 shows the values of A that correspond to the measured $\bar{W}_{\mathrm{p}}(z)$ values plotted in the right-hand panel. We measure the clustering amplitude of quasars at $\sim 25 h^{-1} \mathrm{kpc}$ from our full KDE-complete sample of 47 confirmed binaries to be $A=24.1$ \pm 3.6 (the pink star in Fig. 8).

Croom et al. (2005) measured a clustering amplitude equivalent to $A \sim 5$ at $z \sim 1.5$ on Mpc scales. The fact that we find a factor of $\sim 4 \times$ stronger amplitude for quasar clustering on $\mathrm{kpc}$ scales than has been found on Mpc scales suggests that, on small scales, quasar clustering climbs rapidly above the dark matter model (Smith et al. 2003) that we use in equation (5). This was interpreted as an 'excess' by Hennawi et al. (2006a) and Myers et al. (2008), perhaps driven by pairs of quasars being fed during galaxy mergers. Hopkins et al. (2007) argued instead that strong quasar clustering on small scales is simply indicative of quasars occupying group-scale or 'merger-prone' environments. More recently, the small-scale clustering of quasars has been modelled using the 'one-halo' term in the HOD (e.g. Kayo \& Oguri 2012; Richardson et al. 2012, 2013). As we argue in Section 4.1, this 'excess' is, in fact, probably closeto-consistent with the amplitude of clustering found for some types of galaxies on small scales.

Our unprecedentedly precise measurements of $\bar{W}_{\mathrm{p}}$ on scales of $\sim 25 h^{-1} \mathrm{kpc}$ allow us to make a first comparison of the evolution of quasar clustering over three orders of magnitude in scale. To do so, we compare our measurements to the empirical description of the evolution of quasar clustering derived by Croom et al. (2005) over scales of $1<s<25 h^{-1} \mathrm{cMpc}$. Using our empirical formalism from equation (8), Croom et al. (2005) found the equivalent of $A(z)=\left[0.53+0.289(1+z)^{2}\right]^{2}$. Our goal is to compare the evolution of the amplitude of quasar clustering on $\mathrm{kpc}$ and $\mathrm{Mpc}$ scales. Because we measure a larger amplitude $(A)$ on kpc scales than is found on Mpc scales, we allow the offset in the Croom et al. (2005) empirical description to float and fit a model of the form $A=\left[c+0.289(1+z)^{2}\right]^{2}$. We find a best fit of $c=2.81 \pm$ 0.31 to our measurements in four slices of redshift over the range $0.43<z<2.26$, which we plot in (both panels of) Fig. 8. We 
find that the evolution of the amplitude of quasar clustering on kpc scales across a wide range of redshift is in reasonable agreement with the overall Mpc-scale empirical description of Croom et al. (2005), once we account for the amplitude offset of a factor of $\sim 4 \times$. The $\chi^{2}$ value of our best fit is 4.2 , which is only rejected at a confidence level of 12 per cent. Based on our admittedly highly empirical model of equation (8), this suggests that the evolution of the amplitude of quasar clustering on the smallest scales can be adequately modelled using descriptions of quasar evolution on $\mathrm{Mpc}$ scales.

\section{SUMMARY AND CONCLUSIONS}

We present by far the largest sample of spectroscopically confirmed binary quasars with proper transverse separations of $17.0 \leq R \leq$ $36.2 h^{-1} \mathrm{kpc}$. Our sample, which is $\sim 6 \times$ larger than any previous homogeneously selected sample on these proper scales, is derived from SDSS imaging over an area corresponding to SDSS DR6. Our quasars are targeted using a KDE technique, and confirmed using long-slit spectroscopy on a range of facilities. We derive a statistically complete subsample of 47 binary quasars with $g<20.85$, which extends across angular scales of $2.9 \operatorname{arcsec}<\Delta \theta<6.3 \operatorname{arcsec}$ and redshifts of $0.43<z<2.26$. This sample is targeted from a parent catalogue that would be equivalent to a full spectroscopic survey of nearly 360000 quasars.

We determine the projected correlation function $\left(\bar{W}_{\mathrm{p}}\right)$ of $0.43<z<2.26$ quasars over proper transverse scales of $17.0 \leq R \leq$ $36.2 h^{-1} \mathrm{kpc}$ in four bins of scale. We find that quasars cluster on $\mathrm{kpc}$ scales far higher than implied by a $\gamma=1.8$ power law, as has been adopted by some authors on Mpc scales (e.g. Porciani et al. 2004). For $r_{0}=5 h^{-1} \mathrm{cMpc}$, we find that a power-law slope of $\gamma=1.97$ \pm 0.03 is therefore required to fit quasar clustering on proper scales of $R \sim 25 h^{-1} \mathrm{kpc}$. This is steeper than what is typically measured for galaxies, but is consistent with some measurements of galaxy clustering, particularly on very small scales and at $z>0.5$. We therefore confirm previous results that suggest that the steep shape of quasar clustering on small scales may be indicative of quasars 'turning on' in galaxy mergers (e.g. Hennawi et al. 2006a; Myers et al. 2008) or of quasars inhabiting group-scale ('merger-prone') environments (e.g. Hopkins et al. 2007). The $\gamma \sim 2$ power law we find is also consistent with results that suggest that quasars require a steeper power-law index than is typical for popular theoretical dark matter density relations (e.g. Moore et al. 1996; Navarro, Frenk \& White 1997). A full modelling of this effect will require an in-depth study of the 'one-halo' term of the HOD (e.g. Kayo \& Oguri 2012; Richardson et al. 2012, 2013), which we reserve for future work.

Our sample of binary quasars is the first that is sufficiently large to study quasar clustering as a function of redshift on proper scales of $R \sim 25 h^{-1} \mathrm{kpc}$. To investigate the evolution of quasar clustering on small scales, we measure the projected quasar correlation function in four bins of redshift over $0.4 \leq z \leq 2.3$ and derive the amplitude of quasar clustering on small scales. We find that, at $z \sim 1.5$, the clustering of quasars substantially exceeds our chosen dark matter model (Smith et al. 2003), and it does so by a factor of about 4 in amplitude as compared to the excess over dark matter on Mpc scales.

We compare the evolution of the amplitude of quasar clustering on proper scales of $R \sim 25 h^{-1} \mathrm{kpc}$ to empirical relationships derived by Croom et al. (2005) on scales of $\sim 10 h^{-1} \mathrm{Mpc}$. Our kpc-scale results cannot rule out descriptions of the evolution of quasar clustering on Mpc-scales, which at its simplest would imply that the dark matter in which quasars are embedded evolves similarly to the baryonic matter over three orders of magnitude in scale. However, our sample size is too small and our modelling is too physically simplistic to formally detect a strong evolution in quasar bias from $z \sim 0.5$ to 2.5 , which leaves open the possibility that how the clustering of quasars evolves with redshift may be a function of scale.

\section{ACKNOWLEDGEMENTS}

SE and ADM were partially supported by the National Science Foundation (NSF) through grant number 1515404. SGD, AAM and MJG acknowledge partial support from NSF grants AST-1313422, AST-1413600 and AST-1518308. We thank the staff of Palomar Observatory for their assistance during our observing runs. Observations reported here were obtained at (1) the MMT Observatory, a joint facility of the Smithsonian Institution and the University of Arizona; (2) the Hale Telescope, Palomar Observatory, as part of a continuing collaboration between the California Institute of Technology, NASA/JPL, Oxford University, Yale University and the National Astronomical Observatories of China; and (3) the Mayall telescope at Kitt Peak National Observatory, National Optical Astronomy Observatory (NOAO Prop. ID: 2008A-0127; PI: Myers), which is operated by the Association of Universities for Research in Astronomy (AURA) under cooperative agreement with the $\mathrm{Na}$ tional Science Foundation. The authors are honoured to be permitted to conduct astronomical research on Iolkam Du'ag (Kitt Peak), a mountain with particular significance to the Tohono O'odham. This work used the facilities of The Advanced Research Computing Center at the University of Wyoming (Advanced Research Computing Center. 2012. Mount Moran: IBM System X cluster. Laramie, WY: University of Wyoming. http://n2t.net/ark:/85786/m4159c).

\section{REFERENCES}

Abazajian K. et al., 2003, AJ, 126, 2081

Adelman-McCarthy J. K. et al., 2008, ApJS, 175, 297

Berlind A. A., Weinberg D. H., 2002, ApJ, 575, 587

Blanton M. R., Lin H., Lupton R. H., Maley F. M., Young N., Zehavi I., Loveday J., 2003, AJ, 125, 2276

Chatterjee S., Degraf C., Richardson J., Zheng Z., Nagai D., Di Matteo T., 2012, MNRAS, 419, 2657

Chatterjee S., Nguyen M. L., Myers A. D., Zheng Z., 2013, ApJ, 779, 147

Coil A. L., Hennawi J. F., Newman J. A., Cooper M. C., Davis M., 2007, ApJ, 654, 115

Coil A. L., Mendez A. J., Eisenstein D. J., Moustakas J., 2016, preprint (arXiv:1609.09090)

Cole S., Kaiser N., 1989, MNRAS, 237, 1127

Crampton D., Cowley A. P., Hickson P., Kindl E., Wagner R. M., Tyson J. A., Gullixson C., 1988, ApJ, 330, 184

Croom S. M., Shanks T., 1996, MNRAS, 281, 893

Croom S. M., Smith R. J., Boyle B. J., Shanks T., Miller L., Outram P. J., Loaring N. S., 2004, MNRAS, 349, 1397

Croom S. M. et al., 2005, MNRAS, 356, 415

Croom S. M. et al., 2009, MNRAS, 399, 1755

Dawson K. S. et al., 2013, AJ, 145, 10

Di Matteo T., Springel V., Hernquist L., 2005, Nature, 433, 604

Djorgovski S., 1991, in Crampton D., ed., ASP Conf. Ser. Vol. 21, The Space Distribution of Quasars. Astron. Soc. Pac., San Francisco, p. 349

Djorgovski S., Spinrad H., 1984, ApJ, 282, L1

Djorgovski S., Perley R., Meylan G., McCarthy P., 1987, ApJ, 321, L17

Eftekharzadeh S. et al., 2015, MNRAS, 453, 2779

Ellison S. L., Patton D. R., Mendel J. T., Scudder J. M., 2011, MNRAS, 418, 2043

Ellison S. L., Mendel J. T., Scudder J. M., Patton D. R., Palmer M. J. D., 2013, MNRAS, 430, 3128 
Fan X. et al., 1999, ApJ, 526, L57

Fan X. et al., 2006, AJ, 131, 1203

Favole G. et al., 2016, MNRAS, 461, 3421

Gehrels N., 1986, ApJ, 303, 336

Green P. J., Myers A. D., Barkhouse W. A., Aldcroft T. L., Trichas M., Richards G. T., Ruiz Á., Hopkins P. F., 2011, ApJ, 743, 81

Gregg M. D., Becker R. H., White R. L., Richards G. T., Chaffee F. H., Fan X., 2002, ApJ, 573, L85

Hagen H.-J., Hopp U., Engels D., Reimers D., 1996, A\&A, 308, L25

Hennawi J. F., Prochaska J. X., 2013, ApJ, 766, 58

Hennawi J. F. et al., 2006a, AJ, 131, 1

Hennawi J. F. et al., 2006b, ApJ, 651, 61

Hennawi J. F. et al., 2010, ApJ, 719, 1672

Hewett P. C., Webster R. L., Harding M. E., Jedrzejewski R. J., Foltz C. B., Chaffee F. H., Irwin M. J., Le Fevre O., 1989, ApJ, 346, L61

Hewett P. C., Foltz C. B., Harding M. E., Lewis G. F., 1998, AJ, 115, 383

Hopkins P. F., Somerville R. S., Hernquist L., Cox T. J., Robertson B., Li Y., 2006, ApJ, 652, 864

Hopkins P. F., Lidz A., Hernquist L., Coil A. L., Myers A. D., Cox T. J., Spergel D. N., 2007, ApJ, 662, 110

Hopkins P. F., Hernquist L., Cox T. J., Kereš D., 2008, ApJS, 175, 356

Inada N. et al., 2008, AJ, 135, 496

Inada N. et al., 2010, AJ, 140, 403

Inada N. et al., 2012, AJ, 143, 119

Jiang N., Wang H., Mo H., Dong X., Wang T., Zhou H., 2016, ApJ, 832, 111

Kaiser N., 1984, ApJ, 284, L9

Kayo I., Oguri M., 2012, MNRAS, 424, 1363

Kochanek C. S., Falco E. E., Muñoz J. A., 1999, ApJ, 510, 590

Lacey C., Cole S., 1993, MNRAS, 262, 627

McCarthy P. J., Dickinson M., Filippenko A. V., Spinrad H., van Breugel W. J. M., 1988, ApJ, 328, L29

McGreer I. D., Eftekharzadeh S., Myers A. D., Fan X., 2016, AJ, 151, 61

Masjedi M. et al., 2006, ApJ, 644, 54

Meylan G., Djorgovski S., 1989, ApJ, 338, L1

Meylan G., Djorgovski S., Weir N., Shaver P., 1990, The Messenger, 59, 47

Miller L., Lopes A. M., Smith R. J., Croom S. M., Boyle B. J., Shanks T., Outram P., 2004, MNRAS, 348, 395

Moore B., Katz N., Lake G., Dressler A., Oemler A., 1996, Nature, 379,613

Mortlock D. J., Webster R. L., Francis P. J., 1999, MNRAS, 309, 836

Muñoz J. A., Falco E. E., Kochanek C. S., Lehár J., Herold L. K., Fletcher A. B., Burke B. F., 1998, ApJ, 492, L9

Myers A. D. et al., 2006, ApJ, 638, 622

Myers A. D., Brunner R. J., Nichol R. C., Richards G. T., Schneider D. P., Bahcall N. A., 2007a, ApJ, 658, 85

Myers A. D., Brunner R. J., Richards G. T., Nichol R. C., Schneider D. P., Bahcall N. A., 2007b, ApJ, 658, 99

Myers A. D., Richards G. T., Brunner R. J., Schneider D. P., Strand N. E., Hall P. B., Blomquist J. A., York D. G., 2008, ApJ, 678, 635

Navarro J. F., Frenk C. S., White S. D. M., 1997, ApJ, 490, 493

Oguri M. et al., 2006, AJ, 132, 999

Oguri M. et al., 2008, AJ, 135, 512

Oguri M. et al., 2012, AJ, 143, 120

Oke J. B., Gunn J. E., 1983, ApJ, 266, 713

Padmanabhan N., White M., Norberg P., Porciani C., 2009, MNRAS, 397, 1862

Pâris I. et al., 2016, A\&A, 597, A79

Peebles P. J. E., 1973, ApJ, 185, 413

Pindor B., Turner E. L., Lupton R. H., Brinkmann J., 2003, AJ, 125, 2325
Pindor B. et al., 2006, AJ, 131, 41

Planck Collaboration XIII, 2016, A\&A, 594, A13

Porciani C., Norberg P., 2006, MNRAS, 371, 1824

Porciani C., Magliocchetti M., Norberg P., 2004, MNRAS, 355, 1010

Prochaska J. X. et al., 2013, ApJ, 776, 136

Prochaska J. X., Lau M. W., Hennawi J. F., 2014, ApJ, 796, 140

Richards G. T. et al., 2002, AJ, 123, 2945

Richards G. T. et al., 2004, ApJS, 155, 257

Richards G. T. et al., 2009, ApJS, 180, 67

Richardson J., Zheng Z., Chatterjee S., Nagai D., Shen Y., 2012, ApJ, 755, 30

Richardson J., Chatterjee S., Zheng Z., Myers A. D., Hickox R., 2013, ApJ, 774,143

Ross N. P. et al., 2009, ApJ, 697, 1634

Schlegel D. J., Finkbeiner D. P., Davis M., 1998, ApJ, 500, 525

Schneider D. P., Schmidt M., Gunn J. E., 1994, AJ, 107, 1245

Schneider D. P. et al., 2000, AJ, 120, 2183

Schneider D. P. et al., 2005, AJ, 130, 367

Schneider D. P. et al., 2007, AJ, 134, 102

Schneider D. P. et al., 2010, AJ, 139, 2360

Shanks T., Boyle B. J., 1994, MNRAS, 271, 753

Shanks T., Fong R., Boyle B. J., Peterson B. A., 1987, MNRAS, 227, 739

Sheinis A. I., Bolte M., Epps H. W., Kibrick R. I., Miller J. S., Radovan M. V., Bigelow B. C., Sutin B. M., 2002, PASP, 114, 851

Shen Y. et al., 2007, AJ, 133, 2222

Shen Y. et al., 2009, ApJ, 697, 1656

Smith R. E. et al., 2003, MNRAS, 341, 1311

Sramek R. A., Weedman D. W., 1978, ApJ, 221, 468

Stoughton C. et al., 2002, AJ, 123, 485

Swanson M. E. C., Tegmark M., Blanton M., Zehavi I., 2008, MNRAS, 385 , 1635

Tegmark M. et al., 2004, ApJ, 606, 702

Watson D. F., Berlind A. A., McBride C. K., Masjedi M., 2010, ApJ, 709, 115

Weedman D. W., Weymann R. J., Green R. F., Heckman T. M., 1982, ApJ, 255, L5

Wetzel A. R., Cohn J. D., White M., 2009, MNRAS, 394, 2182

White M. et al., 2012, MNRAS, 424, 933

Wyithe J. S. B., Loeb A., 2005, ApJ, 621, 95

Zehavi I. et al., 2011, ApJ, 736, 59

Zhai Z. et al., 2016, preprint (arXiv:1607.05383)

Zhdanov V. I., Surdej J., 2001, A\&A, 372, 1

\section{SUPPORTING INFORMATION}

Supplementary data are available at MNRAS online.

Table 3. Normalized distribution of the spectroscopic redshifts of quasars in our parent sample of candidates.

Table 5. Candidate quasar pairs drawn from our parent sample.

Please note: Oxford University Press is not responsible for the content or functionality of any supporting materials supplied by the authors. Any queries (other than missing material) should be directed to the corresponding author for this article.

This paper has been typeset from a $\mathrm{T}_{\mathrm{E}} \mathrm{X} / \mathrm{L} \mathrm{T} \mathrm{E} \mathrm{X}$ file prepared by the author. 